\title{
Increasing efficiency of nuclear fuel using burnable absorbers
}

\author{
M. Lovecký*, J. Závorka, J. Jiřičková, R. Škoda \\ Regional Innovation Centre for Electrical Engineering, University of West Bohemia, Univerzitní 8, 30614 Plzeň, Czech Republic
}

\section{A R T I C L E I N F O}

\section{Keywords:}

Nuclear fuel

Burnable absorber

Reactivity excess

Depletion

$\mathrm{U}_{\mathrm{W}} \mathrm{B}_{1}$

\begin{abstract}
A B S T R A C T
The paper describes the development, validation, and use of $\mathrm{U}_{\mathrm{W}} \mathrm{B}_{1}$ code intended for fast calculation of nuclear fuel depletion in burnable absorber research. The degree of effectiveness of burnable absorbers in the form of natural abundance elements, nuclides, and their combinations are compared on metric evaluating characteristic properties of burnable absorbers; namely the initial reactivity compensation, residual poisoning minimization, and the influence of the fuel reactivity for higher fuel burnups. The following materials suitable for burnable absorbers are recommended: $\mathrm{Li}, \mathrm{Pa}$, Ir, In and Re, as well as perspective of Li-6 enrichment; beside previously commonly studied boron and rare earth metals. The research results are intended for the application in other technical-economical analyses of the selection of added materials in the nuclear fuel for increasing the fuel efficiency and nuclear safety.
\end{abstract}

\section{Introduction}

Most nuclear power reactors use $\mathrm{UO}_{2}$ fuel enriched from 3 to $5 \mathrm{wt} \%$ U-235 (Glasstone and Sesonske, 1994). Utilizing higher enrichment allows for longer reactor cycles (Ozer and Edsinger, 2001), the specific value of the enrichment is the result of technical and cost analysis (Almenas and Lee, 1992). Increasing demands on nuclear safety causes further decrease of fuel costs. Therefore, it could be feasible to introduce nuclear fuel with enrichment above $5 \mathrm{wt} \%$ of U-235 because the legislative enrichment limit for non-proliferation is $20 \mathrm{wt} \%$ of $\mathrm{U}$ 235 (10 CFR 50.64). Higher enriched fuel could be seen as too reactive at the beginning of the irradiation. Hence, the excess reactivity has to be compensated.

\subsection{Reactor long-term regulation}

Nuclear fuel reactivity describes the ability to maintain a fission chain reaction. Burnable absorbers compensate for the initial excess of reactivity by absorbing neutrons and thus lowering the reaction rates on uranium fuel. The concentration of burnable absorbers decreases during reactor operation and the fuel reactivity reaches an ideal constant rate (Oka, 2014). The uranium nuclei, that have not undergone fission at the beginning of the irradiation, react with neutrons at further stages of the irradiation. Therefore, reactor operation could be extended. The initial excess of reactivity can be compensated by absorbing additives in the fuel. Absorbers, with high absorption cross section and low absorbing reaction products, have the ability to continuously decrease the absorption bounded by the additive material and can be referred to as good burnable absorbers (Yoo et al., 2017).

Nowadays, long-term regulation of light water reactor (LWR) is commonly performed with boric acid in the coolant and burnable absorbers in the fuel. The maximum content of boric acid can be limited by the requirement of a negative temperature reactivity coefficient (Fadaei, 2011). Therefore, burnable absorbers also have an impact on nuclear safety. Boron, gadolinium, and to a small extent europium (Talamo, 2010) and erbium (Fedosov, 2018) are currently the only materials used in power nuclear reactor operation serving as burnable absorbers. Main form is the integral part of the fuel matrix or a thin layer applied to the outer surface of the fuel pellet. For higher fuel enrichment, the use of these burnable absorbers is not optimal because they burn rather quickly (Bernard and Santamarina, 2016).

\subsection{Burnable absorber selection}

A complete burnable absorber design is a part of complex analysis (Hales et al., 2015). The main reason to deploy burnable absorbers is neutronics properties. However, the additive to the fuel can be also useful from thermal, thermomechanical, and other viewpoints (Karoutas et al., 2018). The additive can increase thermal conductivity and lower fuel operating temperatures, or possibly increase pellet fragmentation and conveniently affect pellet-cladding interactions and stress corrosion cracking (Che et al., 2018).

The aim of the research is to design materials for future burnable absorbers primarily based on neutronics calculations. There are over 80

\footnotetext{
* Corresponding author.

E-mail address: lovecky@rice.zcu.cz (M. Lovecký).
} 
naturally abundant elements, forming more than 400 nuclides; nuclear reactions can further artificially create more than 3000 unstable nuclides. The available literature deals with calculations with several elements mainly of natural composition. Calculations for elements, nuclides and their combinations for different types of nuclear reactors were analyzed for the possibilities of their use in the design of nuclear fuel. A new engineering metric is proposed, the efficiency of burning absorbers can be estimated to serve for further technical and cost analysis.

Due to the high number of calculations in the parametric study it is not feasible to use standard time-consuming calculation codes. Therefore, a brand new $\mathrm{U}_{\mathrm{W}} \mathrm{B}_{1}$ calculation code has been developed. The code addresses Bateman equations to isotopic changes of nuclear fuel and the transport equation for calculating the multiplication factor and the density of neutron flux in nuclear fuel in the new 2sPC depletion scheme. The calculation code was developed to rapidly calculate nuclear fuel depletion with sufficient accuracy, which is achieved by simplifications in areas with little influence on the efficiency of burnable absorbers.

\section{Current burnable absorbers}

Burnable absorber (BA) research can be divided into several key areas interacting with one another:

- selection of BA material,

- experimental verification of BA properties (e.g., manufacturing, mechanical and temperature characteristics),

- design in the fuel rod (discrete, integral),

- design in the fuel assembly (BA weight fraction, number of rods with BA, enrichment),

- core loading optimization (achieving safety limits during the campaign).

The research combines both theoretical calculations for design properties and experimental works for manufacturing and operational verification. Previously mentioned research areas are interconnected and can influence one another.

Currently, BAs are used in the form of B, Er, Eu and Gd compounds. The 1960 Russian summary (Volkov et al., 1961) describes the benefits and possible ways to use BA in the reactors. B, Hf, Eu, Gd, Sm, Cd and $\mathrm{Hg}$ are also listed as suitable materials. Studies devoted to various BA materials often choose rare earth elements. In a next study (Asou and Porta, 1997) rare earth elements Gd, Sm, Er, Eu, Dy are considered. Placing BA in the reflector area of the graphite moderated reactor is the subject of a Dutch study (Van Dam, 2000). B-10, Cd-113, Sm-149, Eu151, Gd-155, Gd-157, Dy-161, Dy-164, Er-167, Hf-177 were chosen as candidate materials. Materials currently studied as promising burnable absorbers are listed in Table 1.

\subsection{Materials in advanced fuel concepts}

Newly developed CANFLEX fuel (Roh et al., 2011) for CANDU reactors will use Dy oxides as a burnable absorber. The Korean study (Kannan and Ganesan, 2010) considers Dy, Er, Eu and Hf in the CANFLEX fuel with the recommendation for using $0.9 \mathrm{wt} \% \mathrm{Er}$.

Er and Eu are suitable BA materials; their use is contradicted by a higher price compared to Gd. Neutronic parameters are more appropriate; Er and Eu burn out at a lower rate than Gd and can be used homogeneously in each fuel rod within an assembly. Er as BA is considered for weapon-grade plutonium fuel pebbles of high temperature reactor (HTR) (Kodochigov et al., 2003) and for mixed oxide (MOX) fuel in graphite RBMK reactors (Balygin et al., 1999), where other materials were studied (Er, Gd, Hf and Lu). The US study (Talamo et al., 2009) of the QUADRISO fuel particles for HTR contemplates BA based on Eu rather than $\mathrm{Er}$ in the earlier studies.
Absorption properties for higher fuel burnup of rapidly burning Gd can be improved by adding a second BA element. In the Czech study (Heraltová, 2015) with VVER-440 fuel, the combination of Gd + Er for a 6-year fuel cycle with fuel up to $7.0 \mathrm{wt} \% \mathrm{U}-235$ is considered. In the Canadian study (Chan et al., 2015), BA in the form of Gd+Eu in the CANDU natural fuel is placed in a thin graphite layer between fuel and coating.

Minor actinides Np-237 and Am-241 can be used as BA for proliferation-resistant fuels (Ronen et al., 2010). These minor actinides increase the proportion of $\mathrm{Pu}-238$ in the plutonium vector and act as BAs.

Nuclear fuels for the purpose of a plutonium weapon program stockpiles disposing are considered in the form of $\mathrm{Pu}$ oxide with low or no $\mathrm{U}$ content. Er is considered as the primary BA, experimental verification was performed in Switzerland (Paratte et al., 1999) and the US (Holliday et al., 2009) with calculation studies. The Russian study (Baranaev et al., 2003) prefers Gd as a BA material.

Another material that can be used as a BA, even though it is not its primary purpose, is Tc-99 as proposed in (Liu et al., 2015). The aim is its transmutation. The homogeneous distribution of Tc-99 in the fuel reduces its reactivity too much, but if placed in the pellet-cladding gap it behaves like a good BA with the ability to reduce the boric acid concentration by as much as $500 \mathrm{ppm}$.

BA can be used as enriched in burnable isotopes instead of using naturally abundant element. B-10 enrichment is industrially matured, typical $90 \%$ enrichment level in boron carbide is considered for sodium fast reactor (SFR) (Kim et al., 2005) and HTR reactors (Obara and Onoe, 2013). Laser enrichment of odd Gd isotopes (Santala et al., 1997) can be used to increase the concentration of burnable isotopes Gd-155 and Gd157 from natural $30 \mathrm{wt} \%$ to $70 \mathrm{wt} \%$.

\subsection{Physical compatibility verification}

Candidate BA materials undergo experimental analysis that verify BA or fuel-BA characteristics, including validation of the calculation codes. Because of low thermal conductivity of gadolinium and fuel temperature limits preventing fuel melting, gadolinium fuel rods are enriched to lower levels than other rods. Placing BA directly into the fuel matrix has the advantage of the most effective reactivity influence. On the other hand, material compatibility is required. Moreover, residual fuel poisoning of BA included in the fuel matrix is another disadvantage that can be diminished by placing BA outside the fuel matrix. In the latter option, BA can be a part of the fuel pin as sprayed coating or part of the fuel assembly as extra burnable rods.

Chemical and thermal compatibility of the BA additive in the fuel was experimentally studied in Korea (Kim et al., 2008) as thermal conductivity of $\mathrm{Gd}_{\mathrm{x}} \mathrm{M}_{\mathrm{y}} \mathrm{O}_{\mathrm{z}}(\mathrm{M}=\mathrm{Ti}, \mathrm{Zr}, \mathrm{Al})$ powder pellets showed the highest values for $\mathrm{GdAlO}_{3}$ and $\mathrm{Gd}_{\mathrm{x}} \mathrm{Ti}_{\mathrm{y}} \mathrm{O}_{\mathrm{z}}$. Another Korean experiment (Rhee et al., 2007) measured the influence of the MnO dopant on the densification of $\mathrm{UO}_{2}$ fuel with $10.0 \mathrm{wt} \% \mathrm{Gd}_{2} \mathrm{O}_{3}$ and showed that $0.1 \mathrm{wt}$ $\% \mathrm{MnO}$ decreased the sintering temperature by more than $100 \mathrm{~K}$. The Japanese thermal expansion study (Une, 1989) and the melting temperature study (Yamanouchi et al., 1988) of $\mathrm{UO}_{2}-\mathrm{Gd}_{2} \mathrm{O}_{3}$ fuel pellets showed a small BA effect in the fuel, even for maximum $2000 \mathrm{~K}$ temperature and maximum content of $10 \mathrm{wt} \% \mathrm{Gd}_{2} \mathrm{O}_{3}$.

\subsection{Fuel assembly design}

After the BA material is selected, optimization of the fuel assemblies follows. One option is a combination of different BA types. When validating the Swiss transport and diffusion code ELCOS (Galperin et al., 1995), a combination of two types of BA rods (Wet Annular Burnable Absorber, WABA and Integral Fuel Burnable Absorber, IFBA) was proposed in one assembly. Boron modeling is shown to be computationally simpler than gadolinium. During the development of the American Reactor IRIS (Franceschini and Petrovic, 2009) with $4.95 \mathrm{wt} \%$ 
Table 1

Burnable absorber materials in currently studied nuclear fuels.

\begin{tabular}{|c|c|c|c|c|}
\hline Material(s) & Spatial placing & Goal & Reactor & Reference \\
\hline $\begin{array}{l}\text { B-10, Cd-113, Sm-149, Eu-151, Gd-155, Gd-157, Dy- } \\
\text { 161, Dy-164, Er-167, Hf-177 }\end{array}$ & reflector area & burnable absorber & HTR & Netherlands (Van Dam, 2000) \\
\hline Dy & fuel matrix & void reactivity coefficient & CANDU & Canada (Roh et al., 2011) \\
\hline Dy, Er, Eu, Hf & fuel matrix & void reactivity coefficient & CANDU & Korea (Kannan and Ganesan, 2010) \\
\hline Er & discrete particles & $\begin{array}{l}\text { disposing Pu stockpiles with } \\
\text { BA }\end{array}$ & HTR & Russia (Kodochigov et al., 2003) \\
\hline Er, Gd, Hf, Lu & fuel matrix & MOX introduction & RMBK & Russia (Balygin et al., 1999) \\
\hline $\mathrm{Eu}$ & coated layer & long-term BA & HTR & USA (Talamo et al., 2009) \\
\hline $\mathrm{Gd}+\mathrm{Er}$ & fuel matrix & combined fast and slow BA & VVER & Czechia (Heraltová, 2015) \\
\hline $\mathrm{Gd}+\mathrm{Eu}$ & fuel matrix & combined fast and slow BA & CANDU & Canada (Chan et al., 2015) \\
\hline Np-237, Am-241 & fuel matrix & proliferation-resistant & LWR & Israel (Ronen et al., 2010) \\
\hline Er & inert matrix fuel & $\begin{array}{l}\text { disposing Pu stockpiles with } \\
\text { BA }\end{array}$ & LWR & $\begin{array}{l}\text { Switzerland (Paratte et al., 1999), USA } \\
\text { (Holliday et al., 2009) }\end{array}$ \\
\hline Gd & inert matrix fuel & $\begin{array}{l}\text { disposing Pu stockpiles with } \\
\text { BA }\end{array}$ & LWR & Russia (Baranaev et al., 2003) \\
\hline Tc-99 & pellet-cladding gap & transmutation & PWR & China (Liu et al., 2015) \\
\hline B-10 enriched & fuel matrix & BA enrichment & SFR & Korea (Kim et al., 2005) \\
\hline B-10 enriched & discrete particles & BA enrichment & HTR & Japan (Obara and Onoe, 2013) \\
\hline Gd-155 + Gd-157 enriched & fuel matrix & BA enrichment & LWR & Finland (Santala et al., 1997) \\
\hline B & discrete rods, fuel matrix & reactivity compensation & LWR & Switzerland (Galperin et al., 1995) \\
\hline Gd & fuel matrix & reactivity compensation & LWR & $\begin{array}{l}\text { Korea (Kim et al., 2008), Japan (Une, 1989), } \\
\text { Japan (Yamanouchi et al., 1988) }\end{array}$ \\
\hline $\mathrm{B}+\mathrm{Er}$ & $\begin{array}{l}\text { sprayed layer on fuel } \\
\text { pellet }+ \text { fuel matrix }\end{array}$ & $\begin{array}{l}\text { long-term } \mathrm{BA}+\text { no residual } \\
\text { reactivity }\end{array}$ & PWR & USA (Franceschini and Petrovic, 2009) \\
\hline B & sprayed layer on rod cladding & more effective spatial placing & LWR & USA (Renk et al., 2010) \\
\hline
\end{tabular}

enrichment, a combined BA in the form of $\mathrm{Zr}$ diboride sprayed layer (IFBA) and Er oxide fuel matrix additive (Integral Burnable Absorber, IBA) is considered for 36-month cycles. Design combines the benefits of erbium (i.e., long-term reactivity compensation, good moderator reactivity coefficient, uniform power profile) and IFBA (i.e., no residual reactivity). The cost model of a combined BA is more advantageous than the standard IFBA. The $\mathrm{U}_{\mathrm{W}} \mathrm{B}_{1}$ fast code can be used to efficiently select a combination of different BA types.

IFBA absorbers are applied as a thin layer to the fuel pellet, another option is spraying BA on the cladding as was demonstrated via pulse ion beam by Sandia Laboratory (Renk et al., 2010). Both Gd and B are not soluble in zirconium, B layer is oxidation resistant. The option of using discrete BA is newly proposed by Koreans as BigT - Burnable absorber integrated guide Thimble ( $Y u$ et al., 2016). The $U_{W} B_{1}$ code allows calculation of depletion with BA located in the fuel and its cladding.

Core loading optimization is a parametric task, each solution can only be considered as the best solution with a given algorithm in the given computing time. It is possible to use a simple linear model of reactivity to evaluate the use of $\mathrm{BA}$, a more complex nonlinear model of pressurized water reactor (PWR) refueling scheme optimization based on perturbation theory in the fuel depletion, genetic algorithm, simulated annealing or various complex mathematical models (Zavaljevski, 1990). By using a new BA homogeneously through the fuel according to the $U_{W} B_{1}$ calculation code, it would be possible to eliminate the need to optimize the BA location in the fuel assembly and to increase the efficiency of the core loading optimization.

\subsection{Neutronics summary}

The basic neutronics parameters of the BAs that were considered in the literature are summarized in Table 2, the data comes from the ENSDF (Tuli, 1996) and ENDF/B-VII.1 (Obložinský, 2011) libraries. In addition to 9 naturally abundant elements (B, Cd, Sm, Eu, Gd, Dy, Er, Hf, Hg), Tc-99 and minor actinides Np-237, Am-241, Am-243 and Cm244 are listed. With the exception of the lighter B nucleus with the reaction $(n, \alpha)$, the dominant absorption reaction $(n, g)$ is present. Minor actinides form heavier isotope after radiation capture and the resulting nuclei can undergo fission. Some BAs have a dominant nuclide that carries the BA properties (B-10, Cd-113), other usually contain several burnable isotopes, the depletion-decay scheme can be very different.

\section{3. $\mathrm{U}_{\mathrm{W}} \mathrm{B}_{1}$ code}

Freely available $\mathrm{U}_{\mathrm{W}} \mathrm{B}_{1}$ nuclear fuel depletion code was developed as a fast fuel depletion code to conduct burnable absorber research. The goal of the research is to optimize new materials as BAs in nuclear fuel. BAs compensate for the initial excess reactivity and consequently allow for lower power peaking factors and longer fuel cycles with higher fuel enrichments. In order to develop new fuel design with burnable absorbers together with increased fuel utilization, computationally very expensive parametric study needs to be performed. Therefore, a fast computational tool is desirable.

The first version of the newly developed $\mathrm{U}_{\mathrm{W}} \mathrm{B}_{1}$ fast nuclear fuel depletion code (Lovecký et al., 2014) significantly reduced calculation time by omitting the solution step for the Boltzmann transport equation. Bateman equations describing inventory changes are solved by a matrix exponential method. The exponential is approximated by a fraction of two polynoms based on the CRAM method (a.k.a., Chebyshev rational approximation method), a relatively new, fast, accurate and stable mathematical approach for dealing with large sparse matrices (Pusa and Leppänen, 2010). However, estimation of multiplication factor during depletion was not sufficiently calculated. Moreover, 1-group effective cross sections for strong absorber models like gadolinium showed disagreement between the $U_{w} B_{1}$ tested code and the Serpent reference code.

Hence, Monte Carlo transport solver for $\mathrm{U}_{\mathrm{W}} \mathrm{B}_{1}$ code was introduced [54] in order to improve code accuracy, remove pre-calculated casedependent data libraries and eliminate constant effective cross section assumption. Two dimensional geometry, fuel pin model, ray-tracing algorithm and ENDF/B-VII.1 data are the main components on the solver. Speed of the Monte Carlo solver is the product of development focus on minimization of CPU utilization at the expense of RAM demands. For light water reactor models, $\mathrm{U}_{\mathrm{W}} \mathrm{B}_{1}$ is on average 10 times faster and have a figure-of-merit (defined as the inverse of the product of the variance and the associated computational time) 5 times higher than MCNP6.

Two-step predictor-corrector method (2sPC) developed for $\mathrm{U}_{\mathrm{W}} \mathrm{B}_{1}$ code is the second feature of the code that makes it faster than other 
Table 2

Basic neutronics parameters of selected burnable absorbers.

\begin{tabular}{|c|c|c|c|c|c|}
\hline $\mathrm{BA}(-)$ & Reaction (-) & Isotope $(-)$ & $\begin{array}{l}\text { Abundance } \\
\text { (at } \%)\end{array}$ & $\begin{array}{l}\text { XS } \\
0.0253 \mathrm{eV} \\
\text { (b) }\end{array}$ & $\begin{array}{l}\text { XS } \\
0.625 \mathrm{eV} \\
\text { (b) }\end{array}$ \\
\hline \multirow[t]{2}{*}{ B } & $(n, \alpha)$ & 10 & 19.80 & 3843.5 & 772.9 \\
\hline & $(\mathrm{n}, \mathrm{g})$ & 11 & 80.20 & 0.0 & 0.0 \\
\hline \multirow[t]{8}{*}{$\mathrm{Cd}$} & $(n, g)$ & 106 & 1.25 & 1.0 & 0.2 \\
\hline & & 108 & 0.89 & 0.9 & 0.2 \\
\hline & & 110 & 12.49 & 11.0 & 2.0 \\
\hline & & 111 & 12.80 & 6.9 & 1.3 \\
\hline & & 112 & 24.13 & 2.2 & 0.4 \\
\hline & & 113 & 12.22 & 19969.3 & 531.3 \\
\hline & & 114 & 28.73 & 0.3 & 0.1 \\
\hline & & 116 & 7.49 & 0.1 & 0.0 \\
\hline \multirow[t]{9}{*}{ Sm } & $(\mathrm{n}, \mathrm{g})$ & 144 & 3.07 & 1.6 & 0.3 \\
\hline & & 147 & 14.99 & 57.0 & 11.8 \\
\hline & & 148 & 11.24 & 2.4 & 0.5 \\
\hline & & 149 & 13.82 & 40511.7 & 596.6 \\
\hline & & 150 & 7.38 & 100.0 & 18.2 \\
\hline & & 151 & - & 15142.2 & 330.9 \\
\hline & & 152 & 26.75 & 206.0 & 48.3 \\
\hline & & 153 & - & 420.1 & 114.3 \\
\hline & & 154 & 22.75 & 8.3 & 1.6 \\
\hline \multirow[t]{7}{*}{$\mathrm{Eu}$} & $(n, g)$ & 151 & 47.81 & 9184.7 & 1599.4 \\
\hline & & 152 & - & 12795.8 & 339.7 \\
\hline & & 153 & 52.19 & 358.0 & 44.9 \\
\hline & & 154 & - & 1353.1 & 132.9 \\
\hline & & 155 & - & 3760.3 & 59340.3 \\
\hline & & 156 & - & 100.0 & 20.1 \\
\hline & & 157 & - & 110.8 & 14.5 \\
\hline \multirow[t]{8}{*}{ Gd } & $(\mathrm{n}, \mathrm{g})$ & 152 & 0.20 & 735.1 & 123.4 \\
\hline & & 153 & - & 22333.9 & 36.8 \\
\hline & & 154 & 2.18 & 85.2 & 10.9 \\
\hline & & 155 & 14.80 & 60737.1 & 124.7 \\
\hline & & 156 & 20.47 & 1.8 & 0.4 \\
\hline & & 157 & 15.65 & 252911.8 & 425.7 \\
\hline & & 158 & 24.84 & 2.2 & 0.5 \\
\hline & & 160 & 21.86 & 1.4 & 0.3 \\
\hline \multirow[t]{7}{*}{ Dy } & $(n, g)$ & 156 & 0.06 & 33.1 & 10.3 \\
\hline & & 158 & 0.10 & 43.1 & 5.4 \\
\hline & & 160 & 2.34 & 56.0 & 16.2 \\
\hline & & 161 & 18.91 & 600.2 & 77.8 \\
\hline & & 162 & 25.51 & 194.0 & 49.1 \\
\hline & & 163 & 24.90 & 123.4 & 50.5 \\
\hline & & 164 & 28.18 & 2653.3 & 309.3 \\
\hline \multirow[t]{6}{*}{ Er } & $(\mathrm{n}, \mathrm{g})$ & 162 & 0.14 & 18.9 & 4.0 \\
\hline & & 164 & 1.60 & 13.0 & 2.6 \\
\hline & & 166 & 33.50 & 16.9 & 3.3 \\
\hline & & 167 & 22.87 & 649.8 & 3545.8 \\
\hline & & 168 & 26.98 & 2.7 & 0.6 \\
\hline & & 170 & 14.91 & 8.9 & 1.8 \\
\hline \multirow[t]{6}{*}{$\mathrm{Hf}$} & $(\mathrm{n}, \mathrm{g})$ & 174 & 0.16 & 549.5 & 64.8 \\
\hline & & 176 & 5.26 & 21.4 & 4.8 \\
\hline & & 177 & 18.60 & 373.7 & 277.2 \\
\hline & & 178 & 27.28 & 83.9 & 19.3 \\
\hline & & 179 & 13.62 & 42.8 & 7.9 \\
\hline & & 180 & 35.08 & 13.1 & 2.6 \\
\hline \multirow[t]{7}{*}{$\mathrm{Hg}$} & $(\mathrm{n}, \mathrm{g})$ & 196 & 0.15 & 3078.2 & 360.8 \\
\hline & & 198 & 9.97 & 2.0 & 0.4 \\
\hline & & 199 & 16.87 & 2149.6 & 264.5 \\
\hline & & 200 & 23.10 & 1.4 & 0.3 \\
\hline & & 201 & 13.18 & 4.9 & 1.0 \\
\hline & & 202 & 29.86 & 5.0 & 1.0 \\
\hline & & 204 & 6.87 & 0.4 & 0.4 \\
\hline Tc-99 & $(\mathrm{n}, \mathrm{g})$ & 99 & - & 20.0 & 4.8 \\
\hline \multirow[t]{2}{*}{ Np-237 } & $(n, g)$ & 237 & - & 175.4 & 64.2 \\
\hline & $(\mathrm{n}, \mathrm{f})$ & 238 & - & 2201.3 & 407.6 \\
\hline \multirow[t]{2}{*}{ Am-241 } & $(\mathrm{n}, \mathrm{g})$ & 241 & - & 684.2 & 983.6 \\
\hline & $(\mathrm{n}, \mathrm{f})$ & 242 & - & 2094.9 & 505.2 \\
\hline Am-243 & $(n, g)$ & 243 & - & 80.4 & 41.9 \\
\hline & $(\mathrm{n}, \mathrm{f})$ & 244 & - & 2300.3 & 559.0 \\
\hline $\mathrm{Cm}-244$ & $(\mathrm{n}, \mathrm{g})$ & 244 & - & 15.2 & 3.2 \\
\hline & $(\mathrm{n}, \mathrm{f})$ & 245 & - & 2054.1 & 200.8 \\
\hline
\end{tabular}

Monte Carlo depletion codes (Dufek et al., 2013). Similarly to Monte Carlo solver, 2sPC method is able to speed-up the calculation approximately 10 times. Hence, overall fuel depletion with $\mathrm{U}_{\mathrm{W}} \mathrm{B}_{1}$ code is expected to be around 100 times faster than with MCNP6 reference code. The idea of $2 \mathrm{sPC}$ method is to change the coupling of transport and burnup solvers by omitting a major fraction of the transport solver callings because the transport solver is orders of magnitude slower than the burnup solver. Both transport and burnup variables are calculated for predicted states and corrected with more precise values as the two parts of the fuel depletion are coupled. Only three transport solver solutions are used (i.e., the initial fuel state, predicted and corrected states for the final burnup state). Effective cross sections are evaluated during fuel depletion by assuming nuclide-based non-linear dependency. Multiplication factors in the depletion steps other than the first and the last one are estimated by neutron production to absorption ratio that is calculated without the need to call the transport solver.

The $\mathrm{U}_{\mathrm{W}} \mathrm{B}_{1}$ nuclear fuel depletion code was the most intensively validated against other codes for the CANDU fuel bundle (Lovecký et al., 2016); the comparison study included WIMS, MCNP6, Serpent, KENO-VI and NEWT codes. The behaviors of all investigated parameters were captured by the code. A slight decrease in the accuracy comes inherently with the methodology and assumptions used in the code. This is expected as the code development focused significantly on the speed of calculation. The lower accuracy is outweighed by the speed-up factor between one to two orders of magnitude.

Further validation was performed on selected depletion cases used in the parametric study. CANDU, VVER and SFR fuel with various enrichments, BA content and placing was analyzed. As burnable absorbers, Am-241 was selected as a representative of minor actinides, B as a typical standard low atomic BA and one rare earth metal $(\mathrm{Eu})$ that is often considered as BA. On average, KENO-VI, MCNP6, NEWT and Serpent neutron multiplication factor agrees with each other within 300 pcm. $U_{W} B_{1}$ has a higher average difference of $800 \mathrm{pcm}$.

The depletion of CANDU fuel is characterized by two effects - the initial transient and the plutonium peak. In the first hours, short-term fission products with high absorption cross sections are rapidly accumulating, which significantly reduces fuel reactivity while providing a temperature rise after fresh fuel is introduced into the core during continuous fuel loading. The plutonium peak is a result of the use of natural uranium and radiation capture at U-238, which gradually increases the reactivity during the first month of the operation to the point where the effect is suppressed by the continuous accumulation of fission products.

$\mathrm{U}_{\mathrm{W}} \mathrm{B}_{1}$ captures both CANDU fuel depletion effects, the difference in neutron multiplication factor between the Serpent reference code and other codes is typically at the level of 0.001 , but rises slightly above 0.01 at some depletion points. It is crucial that the difference in multiplication factor between $\mathrm{U}_{\mathrm{W}} \mathrm{B}_{1}$ and the reference code is the same for fuel without a burnable absorber as well as for a different BA fuel. Because this difference is driving the parametric study, the predictions by $\mathrm{U}_{\mathrm{W}} \mathrm{B}_{1}$ code are acceptable.

The depletion of VVER and SFR fuels are predicted by $\mathrm{U}_{\mathrm{W}} \mathrm{B}_{1}$ in a similar way as with the CANDU fuel, differences between the $\mathrm{U}_{\mathrm{W}} \mathrm{B}_{1}$ code and the reference code are as high as 0.02 with negligible dependency of the burnup and BA type. Unlike CANDU fuel, plutonium peaks do not occur in enriched uranium.

\section{Input variables for the parametric study}

CANDU heavy-water moderated fuel, VVER light water reactor fuel and SFR fast reactor fuel were selected for the parametric study. The calculation results for VVER fuel can be qualitatively accepted for Western PWR types. Similarly, a good BA for the fast-cooled sodium reactor, with slight modifications, will be suitable for other types of fast reactors.

In addition to the expected fuel enrichment $0.71 \mathrm{wt} \% \mathrm{U}-235$ for natural uranium of HWR fuel and $5.0 \mathrm{wt} \% \mathrm{U}-235$ for LWR fuel, an increase in fuel enrichment was also considered. The enrichment values 
used were of $0.71,0.8,1.0$ and $2.0 \mathrm{wt} \% \mathrm{U}-235$ for CANDU and 5.0, 6.0, 8.0 and $10.0 \mathrm{wt} \%$ for VVER fuel. Together with the fast reactor enrichment of $20.0 \mathrm{wt} \% \mathrm{U}-235$, the choice covers legislatively allowed $20 \%$ uranium enrichment for LEU fuels.

A total of 9 fuel variants are defined for 3 types of geometries, differences between fuels of the same geometry type were not considered, e.g., CANDU fuel specific power was chosen $32.908 \mathrm{MW} / \mathrm{MTU}$ regardless of enrichment, similarly $40 \mathrm{MW} / \mathrm{MTU}$ for VVER fuel and a typical average boron concentration $600 \mathrm{ppm}$ in the coolant was considered for all calculated variants. The detailed geometry of fuels is consistent with the currently operated CANDU fuel bundle with 37 elements and VVER-1000 fuel assembly.

CANDU fuel depletion has been calculated to a final burnup 21,000 MWd/MTU, VVER fuel to 50,000 MWd/MTU, and SFR fuel the final burnup of 150,000 MWd/MTU in order to cover the burnup that can be influenced by the BA. The number of depletion time steps was 43,44 and 75 respectively with a finer division at the beginning of the depletion to capture significant changes. Time step lengths varied from 40 MWd/MTU at the beginning of the depletion to a maximum step of $2500 \mathrm{MWd} / \mathrm{MTU}$ for higher burnups. In the case of CANDU fuel (with the smallest burnup), the time steps were chosen three times finer than in the case of LWR and SFR.

Higher fuel enrichments require higher initial reactivity compensation, BA content is higher and some materials do not have sufficient absorption capability to achieve the chosen level of initial reactivity compensation even at a very high BA weight. BA weight fraction of $50 \mathrm{wt} \%$ was set as a maximum BA fraction.

Enhanced CANDU and VVER fuel enrichment brings demand for new BA materials because existing materials are at the limit of their potential. In CANDU fuel, no BA is currently in use, and the new designs are based on Gd + Eu combination homogeneously in all fuel rods. On the other hand, Gd in VVER is mixed heterogeneously in the fuel assembly, only to a few fuel rods. The current BA content of up to $8.0 \mathrm{wt} \%$ $\mathrm{Gd}_{2} \mathrm{O}_{3}$ already has a significant effect on the thermo-mechanical properties of fuel and cannot be increased. Moreover, Gd depletes too rapidly.

The spatial location of the BA was considered homogeneously in the fuel or its cladding. Heterogeneous placing in selected rods was not considered. The reason for this is that the parametric study will provide a comparison of materials as BA in a form useable for further analysis of the design of fuel design. Examples of such an analysis are additives to increase thermal conductivity (Ševeček et al., 2018a) or accident tolerant fuel concepts that include both fuel and the cladding design (Ševeček et al., 2018b). These assays are based on the assumption that the material will be present in all fuel streams.

The initial reactivity compensation was selected for three levels; compensating for $25 \%$ of the initial excess reactivity, $50 \%$ of the excess, and compensation using $1.0 \mathrm{wt} \%$ BA (labeled as rc25, rc50 and wt01). The first option was chosen based on the expected reduction in the initial excess reactivity for the current CANDU and VVER fuels - $25 \%$ compensation of the initial excess reactivity reduces the multiplication factor to the CANDU initial transition rate and also represents the normal reduction in reactivity for VVER fuel that is less sensitive to the exact choice of initial compensation due to the refueling scheme. The last option of $1.0 \mathrm{wt} \% \mathrm{BA}$ was chosen to compare elements and nuclides with low absorption capabilities that reduce reactivity by less than $25 \%$ of the initial excess.

In order to provide a complete comprehensive study, BA materials were selected from all naturally occurring elements and all nuclides in the ENDF/B-VII.1 nuclear data library, a total of 84 elements (with the exception of short lived radioactive elements 43, 61 and 84-89) and 423 nuclides from $\mathrm{H}-1$ to $\mathrm{Fm}-255$. The parametric study was prepared and executed in the following 4 phases:

1. Determination of BA content to compensate for $25 \%$ and $50 \%$ of the initial excess of reactivity based on criticality calculations for all
Table 3

Number of calculation cases of phase 2 parametric study.

\begin{tabular}{llllllll}
\hline \multirow{2}{*}{ Fuel } & BA type & \multicolumn{2}{l}{ rc25 $(25 \% \rho)$} & rc50 $(50 \% \rho)$ & wt01 (1.0 wt\% BA) \\
\cline { 3 - 8 } & & fuel & clad & fuel & clad & fuel & clad \\
\hline CANDU-0.71 & element & 77 & 55 & 53 & 29 & 84 & 84 \\
CANDU-0.8 & element & 75 & 54 & 42 & 25 & 84 & 84 \\
CANDU-1.0 & element & 64 & 41 & 39 & 21 & 84 & 84 \\
CANDU-2.0 & element & 45 & 25 & 23 & 9 & 84 & 84 \\
VVER-5.0 & element & 47 & 34 & 27 & 22 & 84 & 84 \\
VVER-6.0 & element & 41 & 33 & 22 & 11 & 84 & 84 \\
VVER-8.0 & element & 38 & 27 & 21 & 16 & 84 & 84 \\
VVER-10.0 & element & 36 & 25 & 20 & 13 & 84 & 84 \\
SFR-20.0 & element & 49 & 20 & 12 & 5 & 84 & 84 \\
CANDU-0.71 & nuclide & 375 & 260 & 269 & 148 & 423 & 423 \\
CANDU-0.8 & nuclide & 356 & 235 & 201 & 115 & 423 & 423 \\
CANDU-1.0 & nuclide & 293 & 168 & 173 & 84 & 423 & 423 \\
CANDU-2.0 & nuclide & 197 & 100 & 120 & 55 & 423 & 423 \\
VVER-5.0 & nuclide & 241 & 161 & 138 & 84 & 423 & 423 \\
VVER-6.0 & nuclide & 210 & 146 & 123 & 77 & 423 & 423 \\
VVER-8.0 & nuclide & 198 & 134 & 105 & 71 & 423 & 423 \\
VVER-10.0 & nuclide & 175 & 125 & 98 & 60 & 423 & 423 \\
SFR-20.0 & nuclide & 210 & 94 & 79 & 35 & 423 & 423 \\
\hline
\end{tabular}

fuels considered, location of BA (fuel, cladding), all elements and nuclides (total of 273,780 variants).

2. Depletion calculation for phase 1 variants where the BA content is below $50 \%$ and for variants with $1.0 \mathrm{wt} \% \mathrm{BA}$ weight fraction. The total number of variants 16,035 was divided among the fuels according to Table 3. Generally, with the increased enrichment and relocation of BA from the fuel region to the cladding, the number of allowable variants decreases.

3. Repeat phase 1 for $50 \%$ compensation, assuming the use of a pair of elements. The weight fraction of the first element from the $25 \%$ phase 1 case is supplemented by determining the weight of the second element so that the total initial reactivity compensation is $50 \%$. Only fuel-fuel and clad-clad variants are considered. The total count was 283,125 variants.

4. Depletion calculation of element pairs for $50 \%$ of the initial excess of reactivity produced the total number of 18,875 variants.

All together, in this study $5,913,815$ cases were compared, which is unique and provides a complete overview of BA. Using the newly developed $\mathrm{U}_{\mathrm{W}} \mathrm{B}_{1}$, the entire set of calculations took only $10,978 \mathrm{~h}$ on an Intel Core i7 processor. Both parallel calculations with $\mathrm{U}_{\mathrm{W}} \mathrm{B}_{1}$ and multiple simultaneous calculations were used.

\section{Parametric study results - absorption properties}

Calculations performed to the reactivity compensation corresponding to $1.0 \mathrm{wt} \%$ BA compared every BA element, even low absorber materials. Absorbing properties of materials and the potential for their use as BA can be evaluated by the ability to compensate for reactivity in their small amounts as the additive in the fuel. Without a more detailed analysis of how fast the absorber depletes, absorption capabilities can be estimated by an effective cross-section for absorption.

The effective cross section for element absorption is compared in Fig. 1 for CANDU-0.71, in Fig. 2 for VVER-5.0 and in Fig. 3 for SFR-20.0 fuel; adjusted for each fuel type by its typical neutron flux density. The figures further compare nuclear densities and macroscopic cross-sections (product of effective cross-section and nuclear density) of all elements. Fig. 4 measures the proportion of the nuclide fraction with the highest effective (microscopic) effective cross-section on the macroscopic effective cross-section.

Nuclear density of light materials, typically Li and B, is significantly higher due to their lower molar mass for a given BA mass fraction in the 


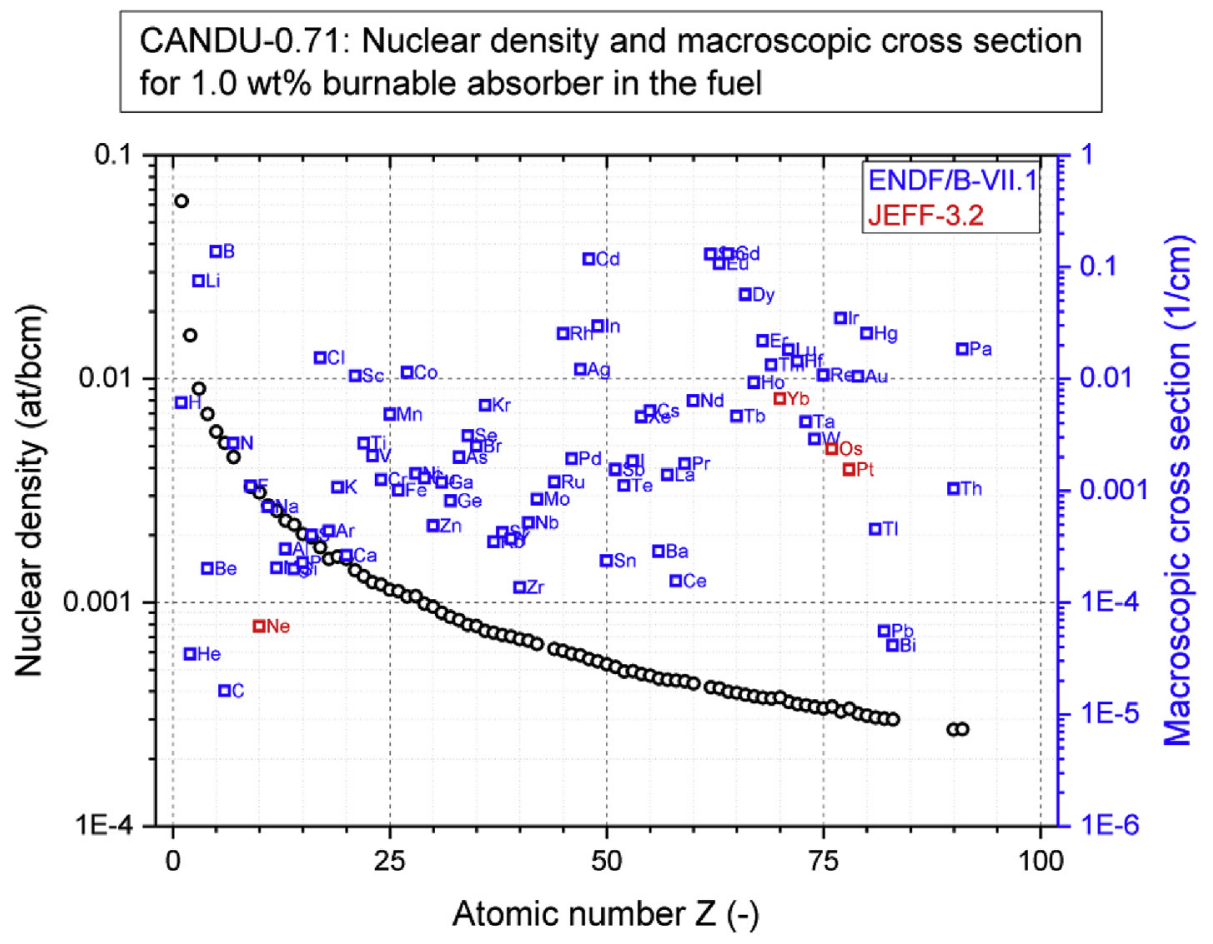

Fig. 1. CANDU fuel -properties of elements as burnable absorber for $1.0 \mathrm{wt} \% \mathrm{BA}$.

fuel. Hence, these materials are good absorbers at lower effective crosssections because the macroscopic effective cross-sections are the variables in the transport equation. High macroscopic cross sections provide moderate elements, typically rare earth elements. The enrichment and the use of the moderator has an influence on the absorption capability, most of the materials have significantly higher thermal cross sections, so SFR fuel efficient cross sections are about an order lower, the differences between CANDU and VVER are lower.

The presence of a high absorbing effective cross section nuclide has a different influence on the overall macroscopic cross-section for a component with a natural composition. For example, He-3, Li-6 and Cd113 nuclides account for more than $99 \%$ of the total absorption capability of their element, but their proportion in nature is only $1.4 \mathrm{E}-4$, 7.6 and $12.2 \%$ respectively. For these elements, the enrichment of absorbing nuclides is a very convenient way to increase their efficiency. Lower enrichment efficiency, although still advantageous, is for B-10 and Gd-157 with a natural content of 19.8 and $15.6 \%$ and a fraction of the macroscopic effective cross-section of 99.9 and $80.7 \%$.

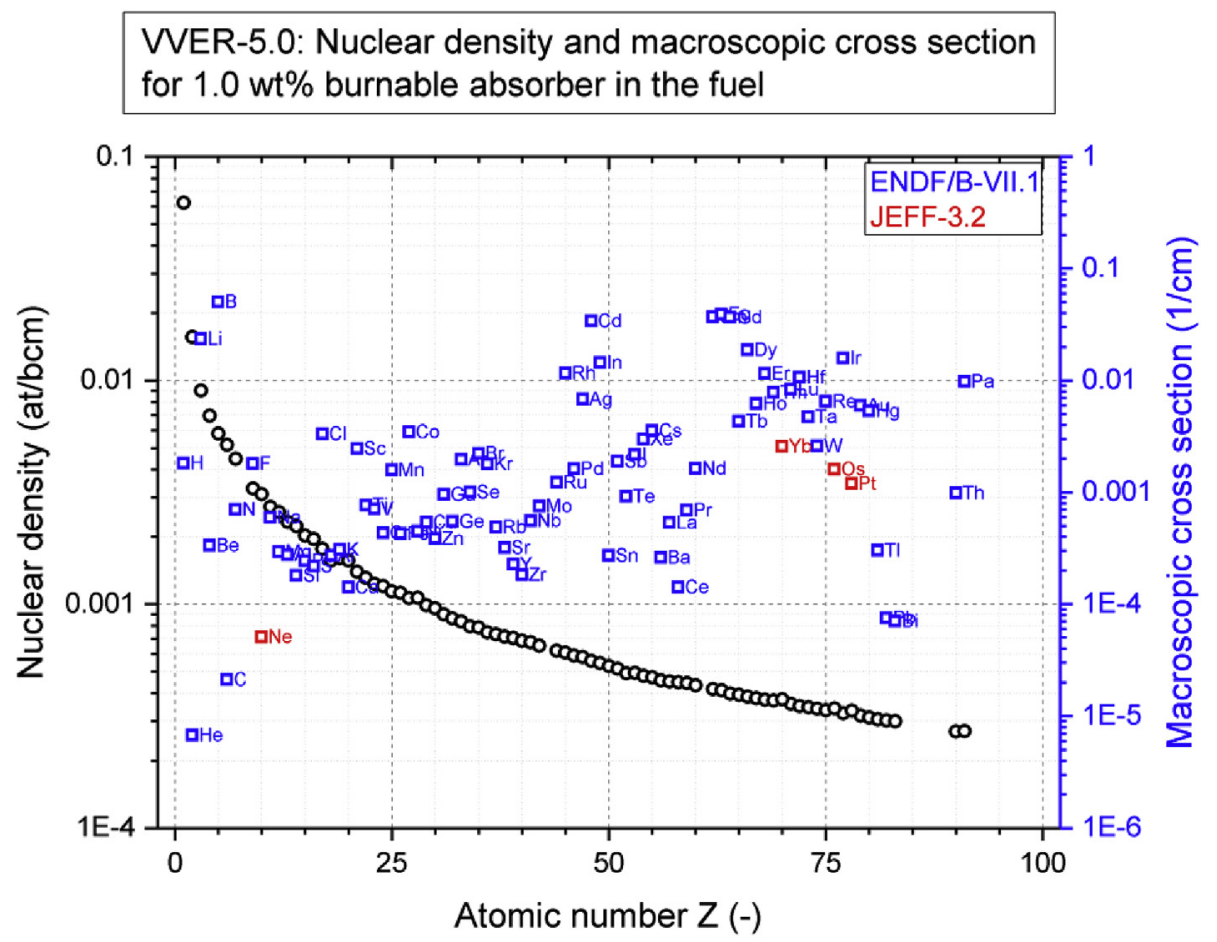

Fig. 2. VVER fuel -properties of elements as burnable absorber for $1.0 \mathrm{wt} \% \mathrm{BA}$. 


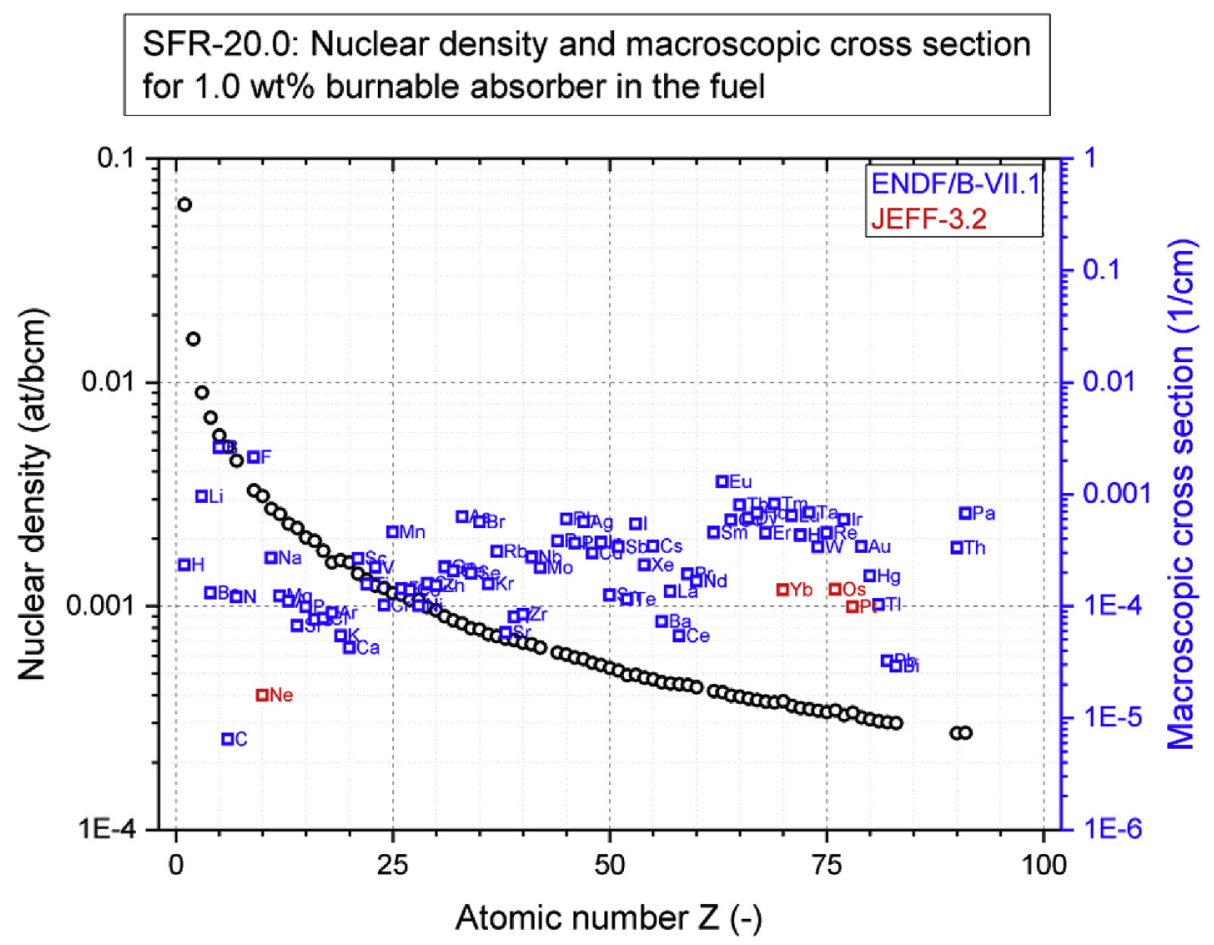

Fig. 3. SFR fuel -properties of elements as burnable absorber for $1.0 \mathrm{wt} \%$ BA.

\section{Parametric study results - material metric MM6}

BAs affect fuel depletion throughout the entire irradiation. Therefore, an engineered metrics has been chosen to quantify the effect of BA. A 6-parameter material metric MM6 has been proposed. The ideal BA maximizes the value of the metric. In contrast to single-parameter metrics, a multi-parameter metric compares BA from multiple independent views, above all, the ability to absorb BA and the ability to deplete faster than the fuel itself is important. Independence of the parameters was analyzed by graphical comparison and statistical tests for 15 different parameters, of which 6 independent parameters for MM6 were selected.

The objective of the 6-parameter metric is to compare materials based on 6 selected properties that have been selected so that they are not correlated. Each of the properties is described by $p_{i}$ parameter as follows:

- Initial reactivity compensation. (N.B. in the case of the preselected

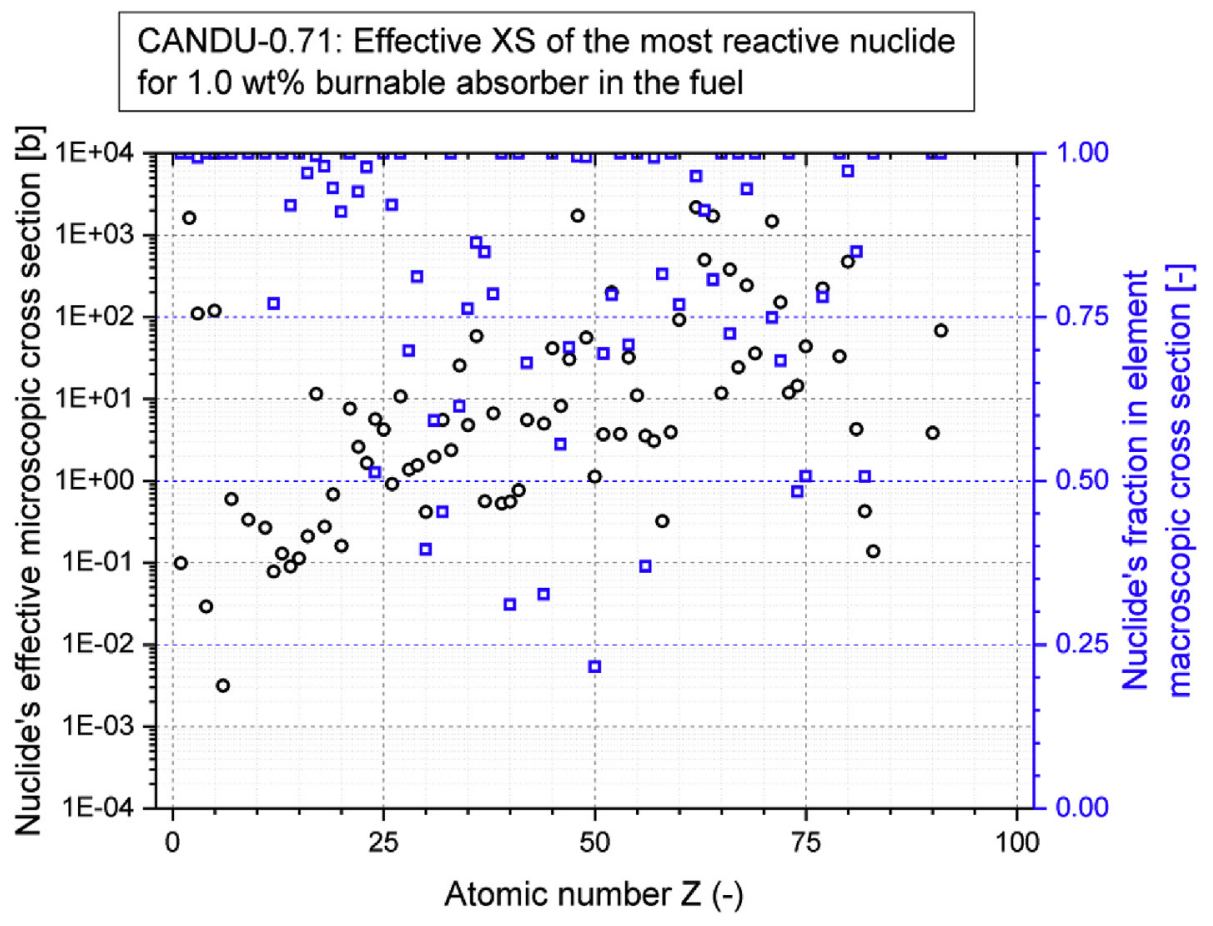

Fig. 4. Absorption fraction of the most reactive nuclide in BA element. 
compensation, it does not apply). The desired BA property is the highest reactivity compensation. For the fresh fuel neutron multiplication factor without $\mathrm{BA}\left(k_{0}\right)$ and with $\mathrm{BA}\left(k_{O, B A}\right)$, the parameter is determined by the relation

$p_{1}=k_{0, B A}-k_{0}$

- Residual poisoning. During depletion, the reactivity bounded by the BA is reduced and in the ideal case, the reactivity at the end of the depletion reaches the same level as in the case of non-poisoned fuel. Minimization of residual poisoning is required, the impact can be alternatively assessed by the reduction of the final burnup with BA. For the maximum expected final burnup $B_{\max }$, the parameter is determined by the relationship

$p_{2}=k_{B \max }-k_{B \max , B A}$

- Fuel reactivity curves with and without BA. The distance is counted as the burnup area between the multiplication factors of both curves. Assuming the parametric study compares cases with fixed initial reactivity compensation, it is required to minimize the area between the two curves to ensure that the fuel reactivity is reduced as slowly as possible. The parameter is given by a relation

$p_{3}=\int_{0}^{B m a x} k_{B A} d B-\int_{0}^{B m a x} k d B$

- Supercritical area during depletion. Similar to the previous parameter, depletion with multiplication factor is integrated, for $p_{4}$ only supercritical part is integrated, and there is no difference between fuel with and without BA. Ideal BA maximizes the area of supercriticality to maximize burnup at $\mathrm{k}=1$ and minimize depletion speed.

$p_{4}=\left.\int_{0}^{B \max } k_{B A} d B\right|_{k>1}$

- BA depletion speed. The ideal BA burns at a constant rate with zero residual poisoning. The best way to estimate the BA depletion speed for typical depletion scenarios of the fuels under consideration is to compare the reactivity bounded by BA at the selected depletion point with the initial reactivity bounded by the BA. A quarter of final burnup was selected to minimize the distance from $50 \%$ of the BA bounded reactivity. When at a constant BA depletion speed, it would be ideal to affect the fuel reactivity to half of its depletion. The $p_{5}$ parameter is given by

$p_{5}=\left|\frac{k_{0.25 B \max }-k_{0.25 B \max , B A}}{k_{0}-k_{0, B A}}-0.5\right|$

- Increasing fuel reactivity with BA during depletion. The goal of the parameter is to penalize undesirable behavior when reactivity increases with $\mathrm{BA}$ at the beginning of the depletion. In the case of homogeneous BA placing, this phenomenon usually does not occur. If so, the effect is evaluated by minimizing the depletion value at the time of reaching the maximum multiplication factor of $k_{\max }$ according to the relationship

$p_{6}=\left.B\right|_{k=k \max }$

The MM6 metric parameters are graphically depicted in Fig. 5. The MM6 metric is defined as the sum of the relative values of $p_{i}$ by the relationship

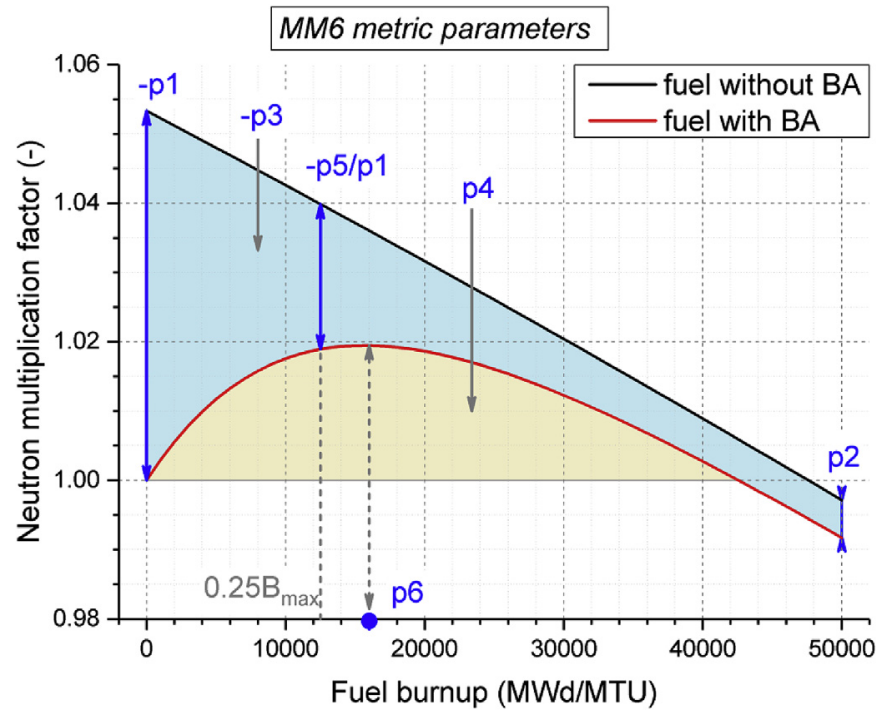

Fig. 5. MM6 engineering metric parameters.

$M M 6=\sum_{i=1}^{6} w_{i}\left(m_{i}-n_{i} \frac{p_{i}-p_{i, \min }}{p_{i, \max }-p_{i, \min }}\right)$

where $w_{i}$ is the relative weight of $p_{i}, p_{i, \min }$ and $p_{i, \max }$ is used to convert the absolute value of $p_{i}$ to a relative value and the integer coefficients $m_{i}$ and $n_{i}$ affect the minimization or maximization of $p_{i}$.

The parameters $m_{i}$ and $n_{i}$ are chosen to maximize the $p_{i}$ parameters by $(0 ;-1)$ or to minimize them by $(1 ; 1)$. After counting the negative $p_{1}$ and $p_{3}$ parameter curves when requesting to maximize $p_{3}$ and $p_{4}$ while minimizing other parameters, the required values are summarized in Table 4.

Weights $w_{i}$ were determined on the basis of a comparison of BA reactivity in the quarter of depletion and residual poisoning. The ideal BA with maximizing the first and minimizing the second parameter is located at the elbow point of the dependency with minimizing the absolute value of the second derivative. For this BA, the relative parameters $p_{i}$ and the weight $w_{i}$ were determined so that for all of these BA all 6 parameters had the same weight in the MM6 metric. The extreme values of the $p_{i}$ relative parameters have been reduced by the logarithmic function.

The values of the $p_{2}$ to $p_{5}$ parameters that affect the overall value of the MM6 metric are compared for VVER fuel in Figs. 6-9. Materials are identified by ZAID number that is defined by the relationship $\mathrm{ZAID}=10000 \mathrm{Z}+10 \mathrm{~A}+\mathrm{m}$, where $\mathrm{Z}$ is atomic number, An atomic mass and $\mathrm{m}$ metastable state. It can be concluded that as $\mathrm{BA}, \mathrm{Li}, \mathrm{B}$, rare earths, Th and Pa materials are well identified in all parameters.

The comparison of the resulting values of MM6 engineering material metric for lowest CANDU enrichment and highest VVER enrichment for $25 \%$ compensation of the initial reactivity for placing BA in the fuel are shown in Fig. 10 and Fig. 11. Placing BA in the cladding results in lower efficiency, but the same qualitative results as for displayed variants. The MM6 for CANDU fuel reaches higher values than for VVER fuel, the lowest values apply to SFR fuel, see Fig. 12 and Fig. 13. Therefore, the

Table 4

MM6 metric variables for relative comparison.

\begin{tabular}{llll}
\hline $\mathrm{i}$ & $\mathrm{w}_{\mathrm{i}}$ & $\mathrm{m}_{\mathrm{i}}$ & $\mathrm{n}_{\mathrm{i}}$ \\
\hline 1 & 0.08 & 1 & 1 \\
2 & 0.33 & 1 & 1 \\
3 & 0.08 & 0 & -1 \\
4 & 0.20 & 0 & -1 \\
5 & 0.24 & 1 & 1 \\
6 & 0.06 & 0 & 1
\end{tabular}




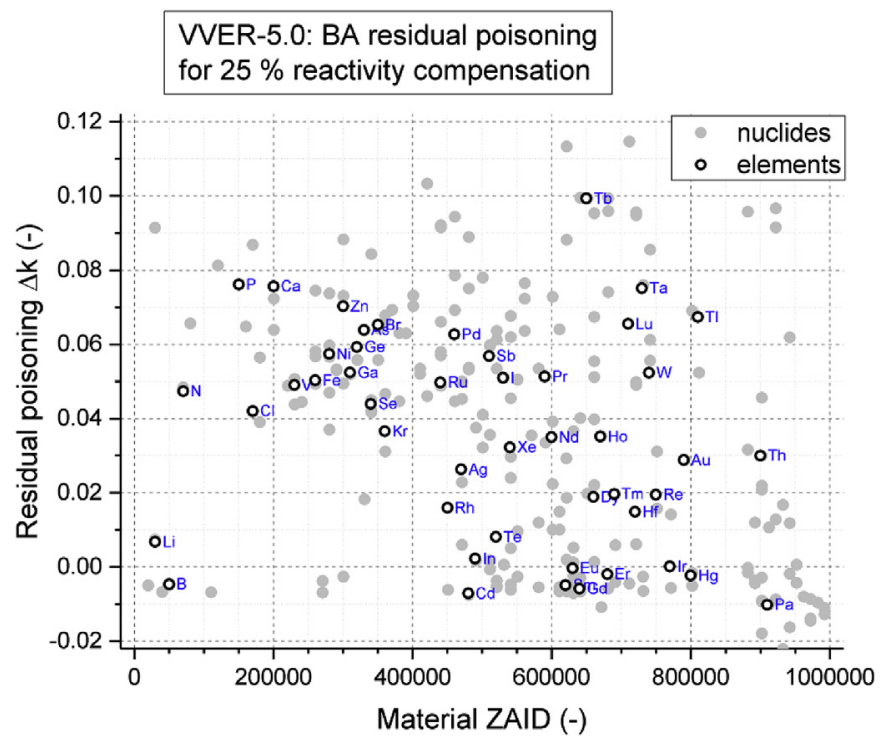

Fig. 6. MM6 parameter $2\left(p_{2}\right)$ for VVER fuel.

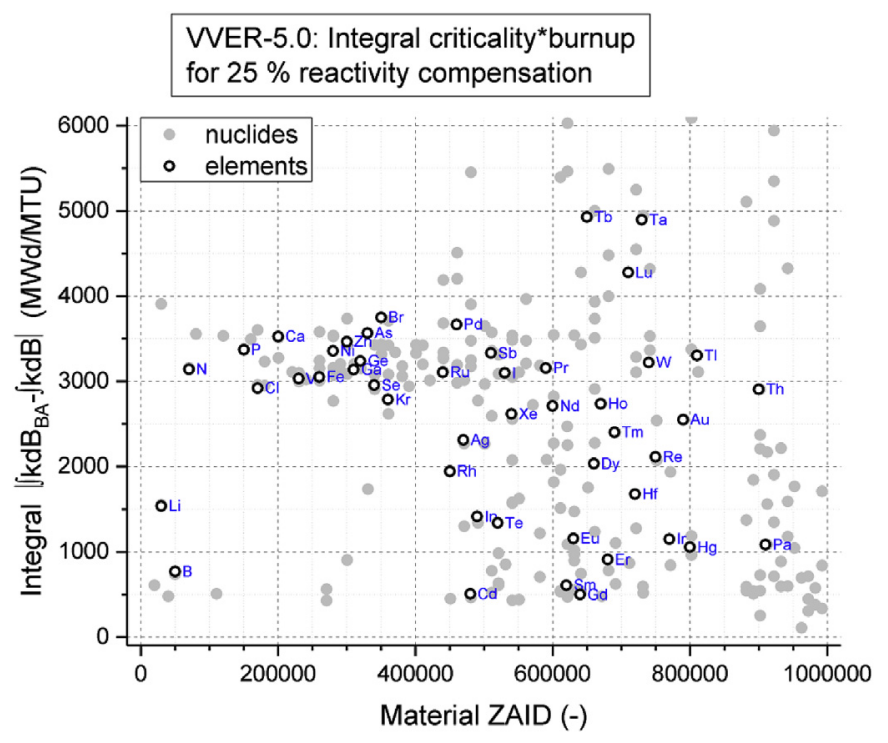

Fig. 7. MM6 parameter $3\left(p_{3}\right)$ for VVER fuel.

widest BA options are available for CANDU fuel. Moreover, the desired BA content is lower than for more enriched fuels.

\section{Parametric study results - material metric MM9}

A comparison of the MM6 metric for variants from phase 2 of parametric study, where elements and nuclides of materials for different fuel types are considered, leads to similar results. For this reason, based on the 6-parameter material metric MM6, another new 9-parameter MM9 metric was defined by adding three new parameters:

- BA location. Fuel and cladding are considered.

- Enrichment and fuel type. 9 types of CANDU-0.71, CANDU-0.8, CANDU-1.0, CANDU-2.0, VVER-5.0, VVER-6.0, VVER-8.0, VVER10.0 and SFR-20.0 fuel types are considered.

- The initial reactivity compensation. Rc25, rc50 and wt01 are considered $-25 \%$ reactivity compensation, $50 \%$ reactivity compensation and $1.0 \mathrm{wt} \% \mathrm{BA}$.

Nine-parametric MM9 metric is determined as the sum of the values

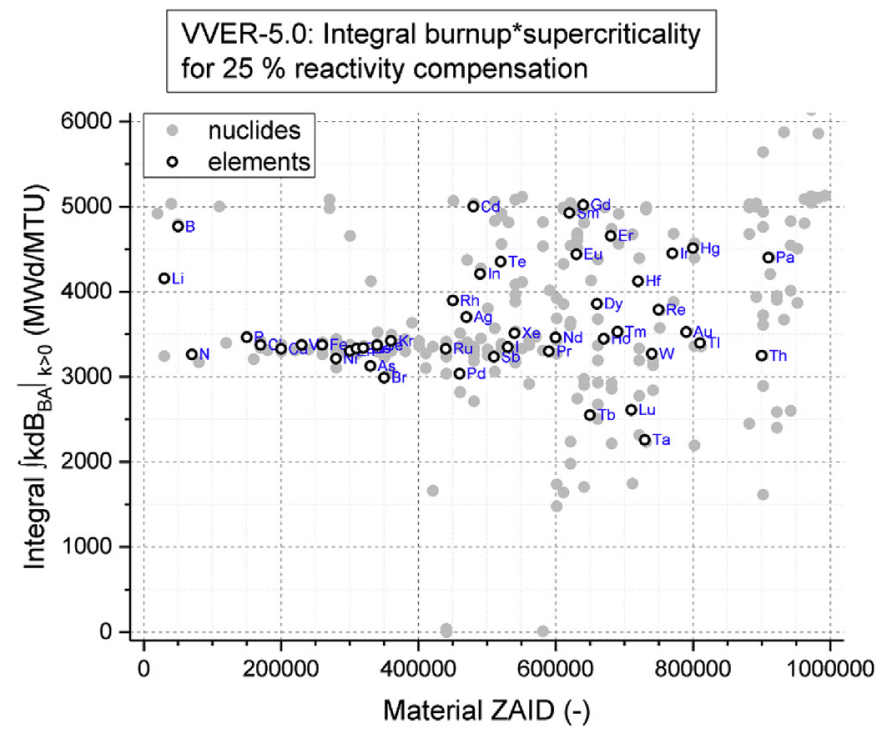

Fig. 8. MM6 parameter $4\left(p_{4}\right)$ for VVER fuel.

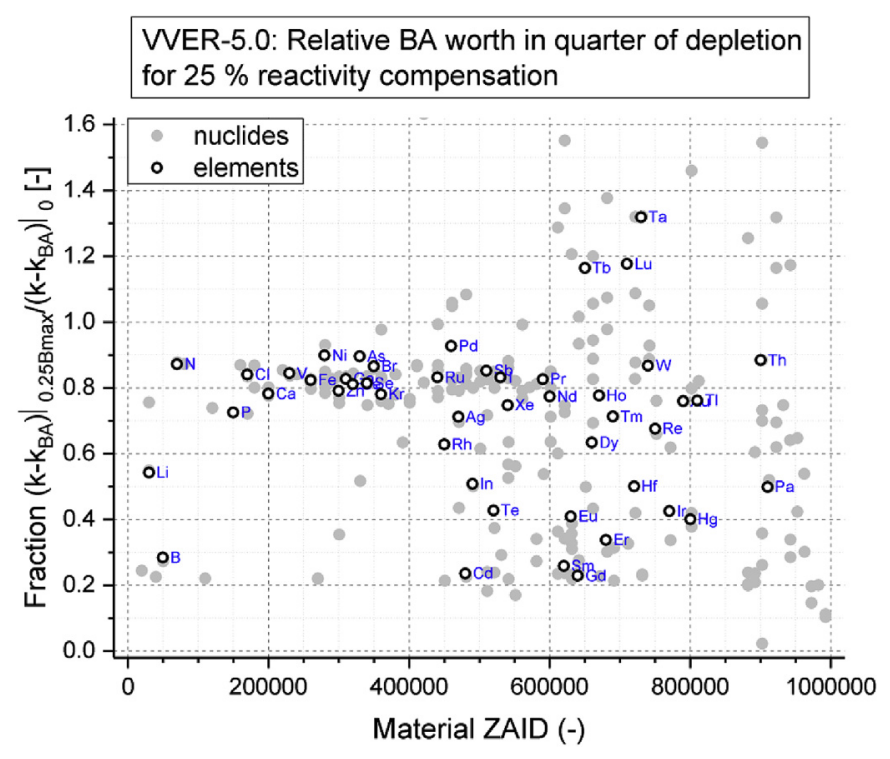

Fig. 9. MM6 parameter $5\left(p_{5}\right)$ for VVER fuel.

of the six-parametric MM6 metric for all considered cases of the previously mentioned 3 parameters.

The resulting values of MM9 metric as the results of a parametric study are summarized in Fig. 14. Selection of the 30 most suitable BA elements and the 30 most suitable BA nuclides in descending order MM9 are listed in Table 5. The optimal element, useable as BA for all the fuel types considered, is Cd, followed by Gd, Sm, Ir, and B. In terms of materials not yet researched, Ir, In, Li and Re can be highlighted. Optimal nuclides, useable as BA, should not have a short half-life. For the three most suitable nuclides the half-life is the tens of days (Pm-148 41d, Be-7 53d, Co-58m 71d), thus they cannot be recommended as BA. From stable nuclides, Cd-113, Gd-157, Eu-155, Er-169, and Sm-149 are part of the optimal BA elements as the optimal BA. As a conclusion of nuclide comparison, it is recommended to enrich the elements with burnable nuclides.

\section{Parametric study results - cluster analysis}

Clustered analysis is a set of statistical methods designed to identify groups of objects based on object characteristics. Objects were chosen 


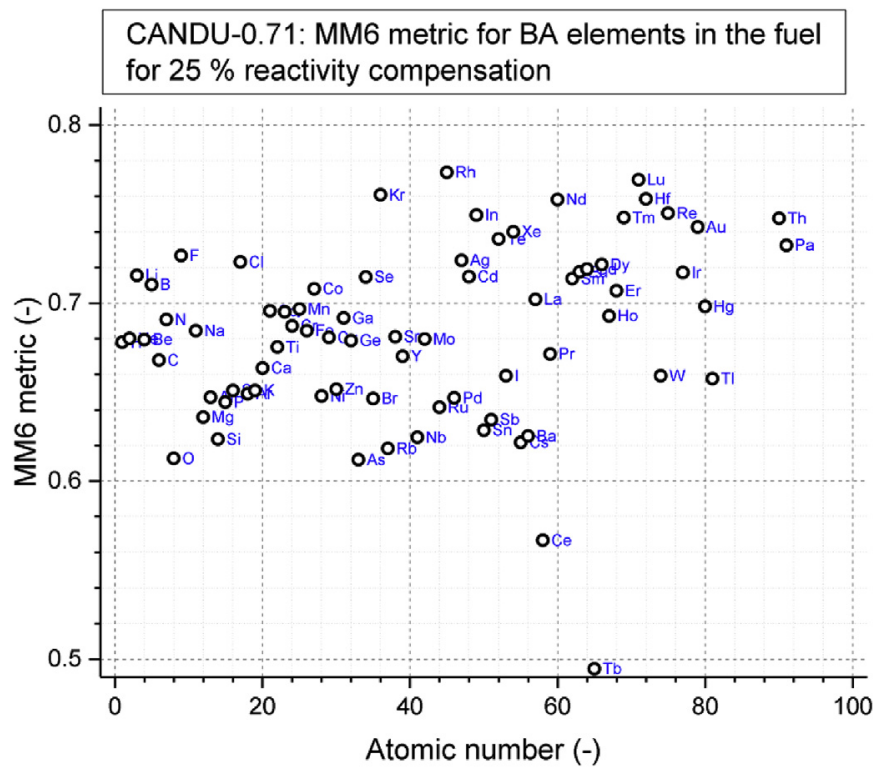

Fig. 10. MM6 metric for BA elements in the fuel for the lowest HWR enrichment.

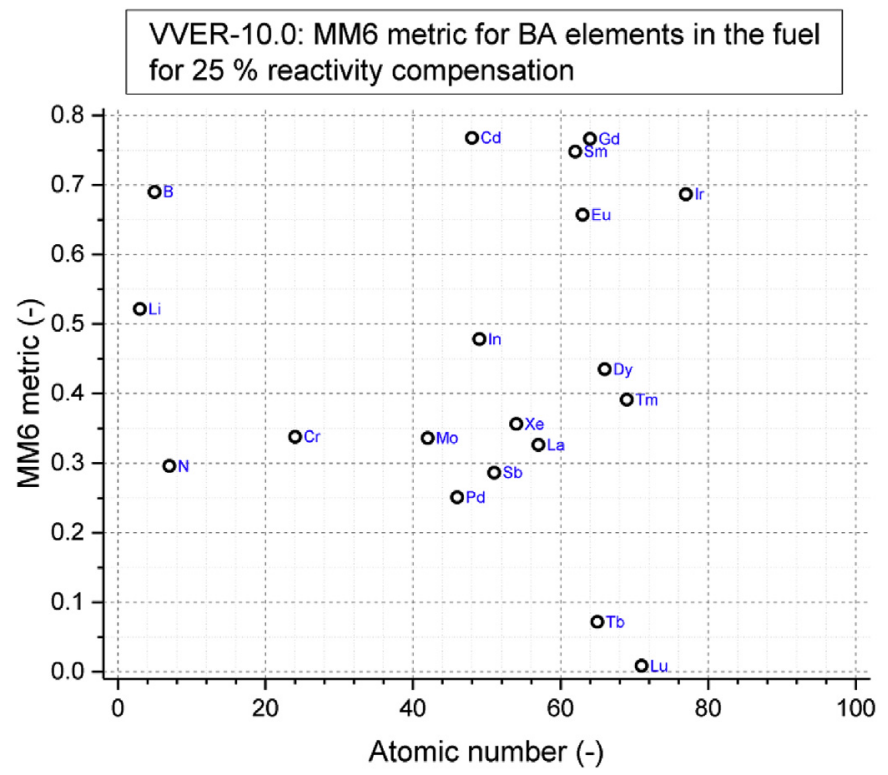

Fig. 11. MM6 metric for BA elements in the fuel for the highest LWR enrichment.

as BA elements in CANDU-0.71 and VVER-5.0 fuels with 25\% initial reactivity compensation, and as object characteristics, $p_{i}$ parameters of the material metric MM6 were chosen.

Cluster analysis computes distance between objects, and it sequentially creates clusters from these objects. The object and the closest cluster of objects are selected and merged into a new cluster at a time. Two-step cluster analysis approach was used. In the first-step, objects are hierarchically clustered, depending on the number of clusters, the cluster distance is determined. Graphically illustrating the dependence of the cluster distance on the number of clusters, a human-hand curve is formed and, according to the elbow rule, the optimum number of clusters is determined. In the second step, non-hierarchical clustering is performed with a given number of clusters.

For CANDU and VVER fuels, BA elements were analyzed by cluster analysis into 4 clusters, SFR fuel BA elements into 3 clusters. By adding two clusters (elements that have no absorption capacity to achieve the

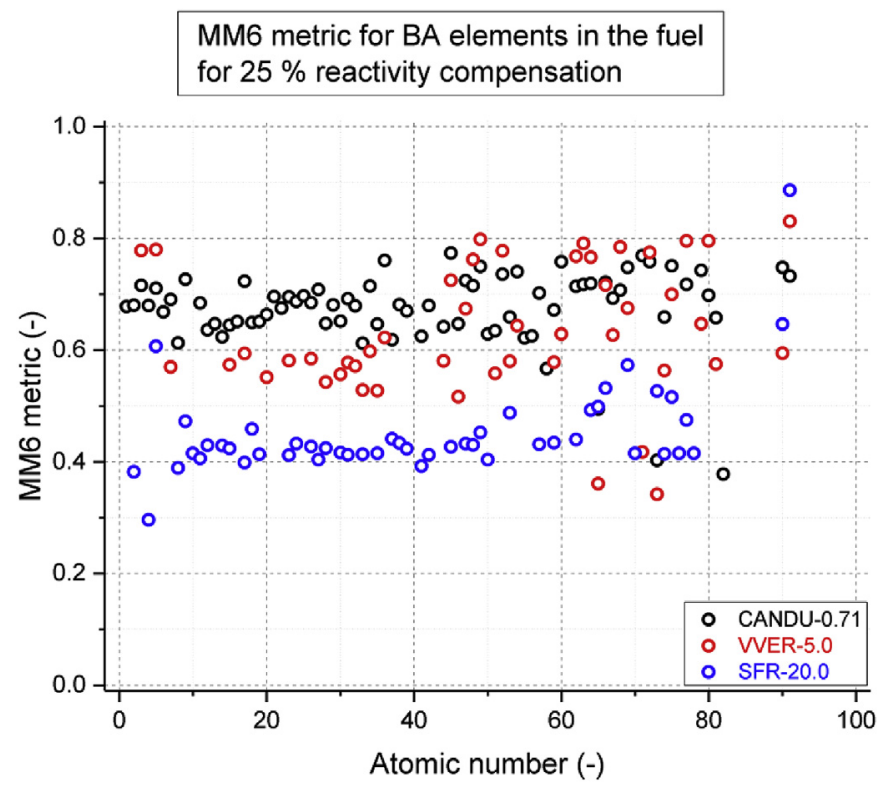

Fig. 12. MM6 metric for - comparison of fuel types for $25 \%$ reactivity compensation.

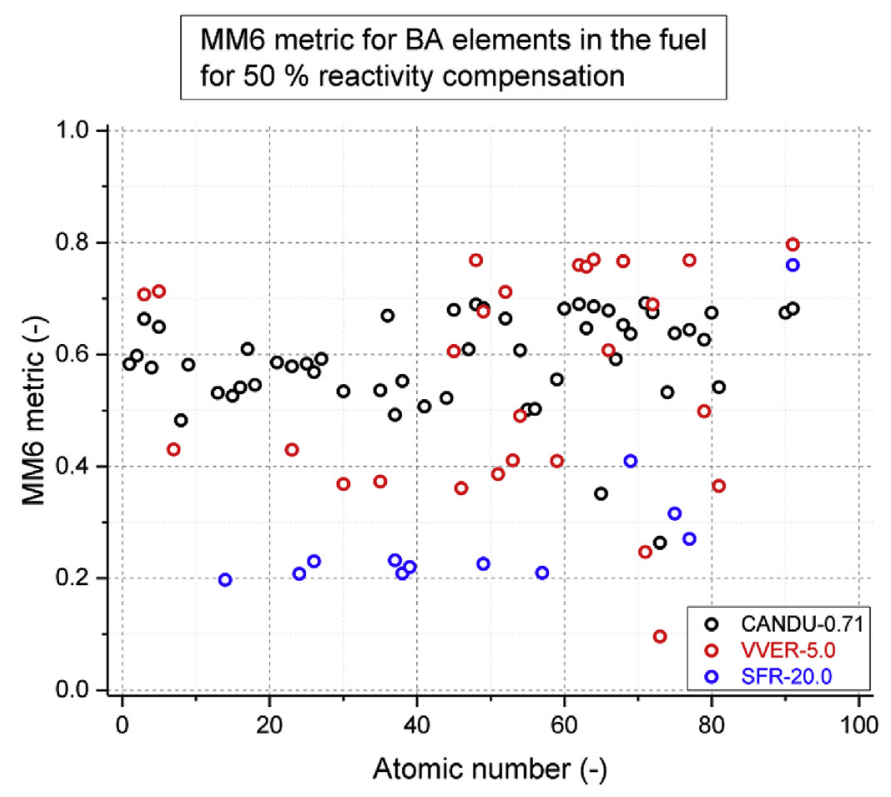

Fig. 13. MM6 metric for - comparison of fuel types for $50 \%$ reactivity compensation.

required reactivity compensation, and radioactive elements), elements were divided into 6 clusters/groups according to Table 6. Based on the average properties of each group and the MM6 metric, elements can be divided into the following groups:

1. Fast burnable absorbers

2. Slow burnable absorbers

3. Absorbers without significant burnable properties

4. Non-burnable elements

5 . Very weak absorbers

6. Radioactive elements

The elements are divided into groups according to the two basic characteristics of BA - the ability to burn and the ability to absorb. Group 4 contains relatively good absorbers, but during the depletion 


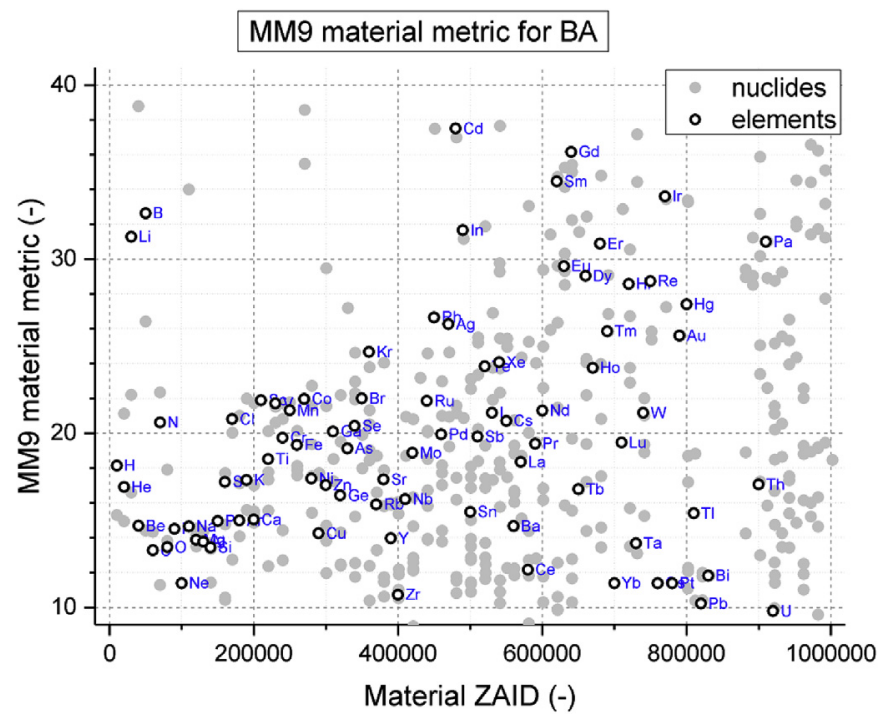

Fig. 14. MM9 metric for the most suitable BA materials.

Table 5

MM9 metric for the most suitable BA materials.

\begin{tabular}{llllllll}
\hline \multicolumn{2}{l}{ Elements } & \multicolumn{7}{l}{ Nuclides } \\
\hline ID & MM9 & ID & MM9 & ID & MM9 & ID & MM9 \\
\hline Cd & \multirow{2}{*}{37.499} & Ag & 26.263 & Pm-148 & 74.699 & Er-167 & 34.786 \\
Gd & 36.160 & Tm & 25.857 & Be-7 & 38.791 & Sm-149 & 34.723 \\
Sm & 34.463 & Au & 25.596 & Co-58m & 38.575 & Am-242 & 34.524 \\
Ir & 33.603 & Kr & 24.655 & Xe-135 & 37.657 & Ta-180 & 34.429 \\
B & 32.632 & Xe & 24.067 & Rh-105 & 37.480 & Bk-245 & 34.417 \\
In & 31.658 & Te & 23.827 & Ta-182 & 37.167 & Eu-154 & 34.145 \\
Li & 31.284 & Ho & 23.753 & Cd-113 & 36.992 & Na-22 & 33.992 \\
Pa & 30.990 & Br & 21.993 & Bk-249 & 36.573 & Ir-191 & 33.433 \\
Er & 30.878 & Co & 21.971 & Cf-250 & 36.235 & Hg-196 & 33.363 \\
Eu & 29.594 & Sc & 21.897 & Th-231 & 35.866 & Hg-199 & 33.285 \\
Dy & 29.031 & Ru & 21.853 & Co-58 & 35.479 & Es-251 & 33.164 \\
Re & 28.735 & V & 21.721 & Gd-157 & 35.420 & Ce-139 & 33.041 \\
Hf & 28.573 & Mn & 21.315 & Eu-155 & 35.259 & Lu-176 & 32.859 \\
Hg & 27.398 & Nd & 21.308 & Es-254 & 35.103 & Th-233 & 32.591 \\
Rh & 26.643 & I & 21.174 & Gd-155 & 35.002 & Dy-164 & 32.441 \\
\hline
\end{tabular}

Table 6

Cluster analysis of elements as burnable absorbers.

\begin{tabular}{|c|c|c|}
\hline Group & CANDU & VVER \\
\hline 1 & Li, B, Cd, Sm, Eu, Gd, Dy, Er, Ir, Hg, Pa & $\mathrm{Cd}, \mathrm{Sm}, \mathrm{Gd}$ \\
\hline \multicolumn{3}{|c|}{, wa } \\
\hline \multirow{3}{*}{3} & $\mathrm{Cl}$, Co, Se, Kr, Rh, Ag, In, Te, Xe, Nd, Tm, Lu, Hf, Re, Au, Th & $\mathrm{Li}, \mathrm{B}, \mathrm{Rh}, \mathrm{In}, \mathrm{Te}, \mathrm{Eu}, \mathrm{Dy}, \mathrm{Er}, \mathrm{Hf}, \mathrm{Re}, \mathrm{Ir}, \mathrm{Hg}, \mathrm{Pa}$ \\
\hline & & $\mathrm{Cl}, \mathrm{V}, \mathrm{Fe}, \mathrm{Ga}, \mathrm{Se}, \mathrm{Kr}, \mathrm{Ru}, \mathrm{Ag}, \mathrm{I}, \mathrm{Xe}, \mathrm{Pr}, \mathrm{Nd}, \mathrm{Ho}, \mathrm{Tm}, \mathrm{Au}, \mathrm{Th}$ \\
\hline & $\begin{array}{l}\text { H, He, Be, C, N, F, Na, Sc, Ti, V, Cr, Mn, Fe, Cu, Ga, Ge, Sr, Y, Mo, La, Pr, } \\
\text { Ho }\end{array}$ & \\
\hline \multicolumn{3}{|c|}{ ( } \\
\hline \multirow{4}{*}{5} & & N, P, Ca, Ni, Zn, Ge, As, Br, Pd, Sb, Tb, Lu, Ta, W, Tl \\
\hline & $\begin{array}{l}\text { O, Mg, Al, Si, P, S, Ar, K, Ca, Ni, Zn, As, Br, Rb, Nb, Ru, Pd, Sn, Sb, I, Cs, } \\
\text { Ba, Ce, Tb, Ta, W, Tl, Pb }\end{array}$ & \\
\hline & $\mathrm{Ne}, \mathrm{Zr}, \mathrm{Yb}, \mathrm{Os}, \mathrm{Pt}, \mathrm{Bi}, \mathrm{U}$ & \\
\hline & & $\begin{array}{l}\mathrm{H}, \mathrm{He}, \mathrm{Be}, \mathrm{C}, \mathrm{O}, \mathrm{F}, \mathrm{Ne}, \mathrm{Na}, \mathrm{Mg}, \mathrm{Al}, \mathrm{Si}, \mathrm{S}, \mathrm{Ar}, \mathrm{K}, \mathrm{Sc}, \mathrm{Ti}, \mathrm{Cr}, \mathrm{Mn}, \mathrm{Co}, \mathrm{Cu}, \mathrm{Rb}, \mathrm{Sr}, \mathrm{Y}, \mathrm{Zr}, \mathrm{Nb}, \mathrm{Mo}, \mathrm{Sn} \text {, } \\
\mathrm{Cs}, \mathrm{Ba}, \mathrm{La}, \mathrm{Ce}, \mathrm{Yb}, \mathrm{Os}, \mathrm{Pt}, \mathrm{Pb}, \mathrm{Bi}, \mathrm{U}\end{array}$ \\
\hline 6 & Tc, Pm, Po, At, Rn, Fr, Ra, Ac & Tc, Pm, Po, At, Rn, Fr, Ra, Ac \\
\hline
\end{tabular}

process the reactivity bounded by the element does not change or increases undesirably.

\section{Conclusions}

A full comprehensive parametric study of materials as burnable absorbers has been evaluated. Elements, nuclides and combinations of elements located in the fuel or its cladding for various enrichment of CANDU, VVER and SFR fuels were compared. The research results are useful for further technical and cost analysis of the selection of nuclear fuel additive materials to enhance its efficiency and safety. Future research supported by experiments has the potential to design new types of nuclear fuel.

The results of the parametric study of materials that can be used as burnable absorbers can be used in other technical and cost analysis because the design of a nuclear fuel design requires an interdisciplinary approach. Any additives in the nuclear fuel or its cladding can also be used as a burnable absorber, it is advisable to optimize these properties. One material can, for example, primarily serve to increase the thermal conductivity of the fuel, but may also have the burnable absorber properties, or may increase safety in accident scenarios. The basic requirement is a chemical compatibility of the additive and the fuel.

For example, Li was determined as one of the prospective materials. Li-6 isotope carries the burnable absorber properties but has a small fraction in natural Li, only 7.6 at\%. Enrichment of Li-6 could be economically advantageous because Li consumption increases due to use for energy storage in batteries and Li enrichment is considered for the fusion reactor blankets. Waste Li-7 with minimal impact on chemical interactions can be used in batteries and reduce the cost of Li enrichment. One of the research directions could therefore be experimental verification of Li-6 enrichment.

The motivation for introducing new burnable absorber materials is the shift of the development of nuclear fuels to higher burnup, accident tolerant fuels and the use of small modular reactors whose nuclear fuel can withstand up to several decades without being replaced, which can only be achieved by using an adequate burnable absorber.

The calculations were performed by the newly developed fast depletion calculation code $\mathrm{U}_{\mathrm{W}} \mathrm{B}_{1}$. The $\mathrm{U}_{\mathrm{W}} \mathrm{B}_{1}$ code for the fast calculation of nuclear fuel depletion is composed of a solver for Bateman's equations using the CRAM method, the Monte Carlo solver for transport equation solution and the $2 \mathrm{sPC}$ depletion scheme to accelerate calculations by the two-step predictor-corrector method. $\mathrm{U}_{\mathrm{W}} \mathrm{B}_{1}$ code speed is 1-2 orders of magnitude higher than standard codes.

The efficiency of the burnable absorbers in the form of elements of natural composition, nuclides and combinations thereof in the fuel or its cladding was compared according to the 6-parameter material 
metric MM6 appreciating the properties of the burnable absorbers, in particular the ability to compensate for the initial excess of reactivity, to minimize residual poisoning and to influence fuel reactivity even for higher fuel burnup. The optimal element, useable as BA for all the considered fuel types, is Cd, followed by Gd, Sm, Ir, and B. In terms of materials not yet researched, Ir, In, Li and Re can be highlighted.

\section{Acknowledgements}

R\&D has been funded by TE01020455 Centre for Advanced Nuclear Technologies (CANUT) project.

\section{References}

10 CFR 50.64. Limitations on the Use of Highly Enriched Uranium (HEU) in Domestic Non-power Reactors.

Almenas, K., Lee, R., 1992. Nuclear Engineering. Springer-Verlag.

Asou, M., Porta, J., 1997. Prospects for poisoning reactor cores of the future. Nucl. Eng. Des. 168, 261-270

Balygin, A.A., Burlakov, E.V., Krayushkin, A.V., Novikov, V.G., Tishkin, Y.A., Fedosov, A.M., 1999. Use of mixed uranium-plutonium fuel with different burnable absorbers in RBMK reactors. Atom. Energy 86, 161-165.

Baranaev, Y.D., Popov, V.V., Sharapov, V.N., Troyanov, V.M., 2003. The feasibility of employing inert matrix ceramic fuels in a Russian light water reactor. J. Nucl. Mater. 319, 154-158.

Bernard, D., Santamarina, A., 2016. Qualification of gadolinium burnable poison: inter pretation of MELUSINE/GEDEON-II spent fuel analysis. Ann. Nucl. Energy 87, 21-33.

Chan, P.K., Paquette, S., Bonin, H.W., 2015. Variation of burnable neutron absorbers in a heavy water-moderated fuel lattice: a potential to improve CANDU reactor operating margins. Nucl. Technol. 191, 1-14.

Che, Y., Pastore, G., Hales, J., Shirvan, K., 2018. Modeling of $\mathrm{Cr}_{2} \mathrm{O}_{3}$-doped $\mathrm{UO}_{2}$ as a nearterm accident tolerant fuel for LWRs using the BISON code. Nucl. Eng. Des. 337, 271-278.

Dufek, J., Kotlyar, D., Shwageraus, E., Leppänen, J., 2013. Numerical stability of the predictor-corrector method in Monte Carlo burnup calculations of critical reactors. Ann. Nucl. Energy 56, 34-38.

Fadaei, A.H., 2011. Investigation of burnable poisons effects in reactor core design. Ann. Nucl. Energy 38, 2238-2246.

Fedosov, A.M., 2018. RBMK uranium-erbium fuel. Atom. Energy 124, 221-226.

Franceschini, F., Petrovic, B., 2009. Fuel with advanced burnable absorbers design for the IRIS reactor core: combined Erbia and IFBA. Ann. Nucl. Energy 36, 1201-1207.

Galperin, A., Grimm, P., Raizes, V., 1995. Modelling and verification of the PWR burnable poison designs by ELCOS code system. Ann. Nucl. Energy 22, 317-325.

Glasstone, S., Sesonske, A., 1994. Nuclear Reactor Engineering. Chapman \& Hall.

Hales, J.D., Tonks, M.R., Gleicher, F.N., Spencer, B.W., Novascone, S.R., Williamson, R.L., Pastore, G., Perez, D.M., 2015. Advanced multiphysics coupling for LWR fuel performance analysis. Ann. Nucl. Energy 85, 98-110.

Heraltová, L., 2015. Possibility of implementation of 6-year fuel cycle at NPP with VVER440 reactor. Nucl. Eng. Des. 295, 40-47.

Holliday, K., Hartmann, T., Czerwinski, K., 2009. Synthesis and characterization of zirconia-magnesia inert matrix fuel: Ce homolog studies. J. Nucl. Mater. 392, 487-493.

Kannan, U., Ganesan, S., 2010. Dysprosium as a resonance absorber and its effect on the coolant void reactivity in Advanced Heavy Water Reactor (AHWR). Ann. Nucl. Energy 37, 270-276.

Karoutas, Z., Brown, J., Atwood, A., Hallstadius, L., Lahoda, E., Ray, S., Bradfute, J., 2018. The maturing of nuclear fuel: past to accident tolerant fuel. Prog. Nucl. Energy $102,68-78$.

Kim, Y.N., Kim, J.K., Park, W.S., 2005. A study on improvement of sodium cooled TRU burner design using burnable poison. Prog. Nucl. Energy 47, 361-368.

Kim, H.S., Joung, Ch. Y., Lee, B.H., Kim, S.H., Sohn, D.S., 2008. Characteristics of $\mathrm{GdxMyOz}(\mathrm{M}=\mathrm{Ti}, \mathrm{Zr}$ or Al) as a burnable absorber. J. Nucl. Mater. 372, 340-349.
Kodochigov, N., Sukharev, Y., Marova, E., Ponomarev-Stepnoy, N., Glushkov, E., Fomichenko, P., 2003. Neutronic features of the GT-MHR reactor. Nucl. Eng. Des. 37, 161-171.

Liu, B., Huang, L., Tu, J., Liu, F., Cao, Q., Jia, R., Li, X., Cai, J., 2015. Technetium transmutation in thin layer coating on PWR fuel rods. Prog. Nucl. Energy 85, 375-383.

Lovecký, M., Piterka, L., Prehradný, J., Škoda, R., 2014. UWB $\mathrm{B}_{1}$ - Fast nuclear fuel depletion code. Ann. Nucl. Energy 71, 333-339.

Lovecký, M., Škoda, R., Hussein, M., Song, J., Chan, P., 2016. The Application of $U_{W} B_{1}$ nuclear fuel depletion Code on a CANDU fuel bundle. Prog. Nucl. Energy 90, $127-139$.

Obara, T., Onoe, T., 2013. Flattening of burnup reactivity in long-life prismatic HTGR by particle type burnable poisons. Ann. Nucl. Energy 57, 216-220.

Obložinský, P., 2011. Special issue on ENDF/B-VII.1 library. Nucl. Data Sheets 112, 2887-3152.

Oka, Y., 2014. Nuclear Reactor Design. Springer.

Ozer, O., Edsinger, K., 2001. Optimum Cycle Length and Discharge Burnup for Nuclear Fuel - A Comprehensive Study for BWRs and PWRs; Phase I; Results Achievable within the 5 Percent Enrichment Limit. Electric Power Research Institute EPRI TR 1003133.

Paratte, J.M., Chawla, R., Früh, R., Joneja, O.P., Pellovni, S., Pralong, C., 1999. Validation efforts for the neutronics of a plutonium-erbium-zirconium oxide inert matrix light water reactor fuel. J. Nucl. Mater. 274, 120-126.

Pusa, M., Leppänen, J., 2010. Computing the matrix exponential in burnup calculations. Nucl. Sci. Eng. 164, 140-150.

Renk, T.J., Sridharan, K., Harrington, S.P., Johnson, A.K., 2010. Incorporation of gadolinium and boron into Zirconium alloy: Surface alloying of immiscible materials using an intense pulsed ion beam. Nucl. Instrum. Methods Phys. Res. B 268, 2666-2678.

Rhee, Y.W., Kim, K.S., Song, K.W., 2007. Densification kinetics of $\mathrm{MnO}$-doped $\mathrm{UO}_{2}-10 \mathrm{wt}$ $\% \mathrm{Gd}_{2} \mathrm{O}_{3}$ compact. Thermochim. Acta 455, 80-85.

Roh, G., Kim, Y., Cho, N.Z., 2011. Improvement of power coefficient by using burnable poison in the CANDU reactor. Nucl. Eng. Des. 241, 1565-1578.

Ronen, Y., Golyand, L., Shwageraus, E., 2010. The potential use of Am-241 as proliferation resistant burnable poison in PWRs. Ann. Nucl. Energy 37, 201-207.

Santala, M.I.K., Daavittila, A.S., Lauranto, H.M., Salomaa, R.R.E., 1997. Odd-isotope enrichment studies of Gd by double resonance laser-ionization for the production of burnable nuclear reactor poison. Appl. Phys. B 64, 339-347.

Ševeček, M., Kubáň, J., Valach, M., Škoda, R., 2018a. Development of high thermal conductivity UO2-Th heterogeneous fuel. Prog. Nucl. Energy 108, 489-496.

Ševeček, M., Gurgen, A., Seshadri, A., Che, Y., Wagih, M., Phillips, B., Champagne, V., Shirvan, K., 2018b. Development of Cr cold spray-coated fuel cladding with enhanced accident tolerance. Nucl. Eng. Technol. 50, 229-236.

Talamo, A., 2010. A novel concept of QUADRISO particles. Part II: utilization for excess reactivity control. Nucl. Eng. Des. 240, 1919-1927.

Talamo, A., Pouchon, M.A., Venneri, F., 2009. Alternative configurations for the QUADRISO fuel design concept. J. Nucl. Mater. 3, 264-266.

Tuli, J.K., 1996. Evaluated nuclear structure data file. Nucl. Instrum. Methods Phys. Res. $369,506-510$.

Une, K., 1989. Thermal Expansion of $\mathrm{UO}_{2}-\mathrm{Gd}_{2} \mathrm{O}_{3}$ fuel pellets. J. Nucl. Sci. Technol. 23, 1020-1022.

Van Dam, H., 2000. Long-term control of excess reactivity by burnable poison in reflector regions. Ann. Nucl. Energy 27, 63-69.

Volkov, V.S., Lukyanov, A.S., Chapkunov, V.V., Shevyakov, V.P., Yamnikov, V.S., 1961. Use of burnable poisons in nuclear reactors. Atom. Energy 11, 109-125.

Yamanouchi, S., Tachibana, T., Tsukui, K., Oguma, M., 1988. Melting Temperature of irradiated $\mathrm{UO}_{2}$ and $\mathrm{UO}_{2}-2 \mathrm{wt} \% \mathrm{Gd}_{2} \mathrm{O}_{3}$ fuel Pellets up to Burnup of about $30 \mathrm{GWd} / \mathrm{tU}$. J. Nucl. Sci. Technol. 25, 528-533.

Yoo, H., Hwang, D.H., Hong, S.G., Shin, H. Ch., 2017. New long-cycle small modular PWR cores using particle type burnable poisons for low boron operation. Nucl. Eng. Des. 314, 173-181.

Yu, H., Yahya, M.S., Kim, Y.A., 2016. A reduced-boron OPR1000 core based on the BigT burnable absorber. Nucl. Eng. Technol. 48, 318-329.

Zavaljevski, N., 1990. Optimization in low leakage PWRs. Ann. Nucl. Energy 17, $217-220$. 